\title{
Ultrawideband Transceiver Design Using Channel Phase Precoding
}

\author{
Yu-Hao Chang, Shang-Ho Tsai, Member, IEEE, Xiaoli Yu, and C.-C. Jay Kuo, Fellow, IEEE
}

\begin{abstract}
A novel transceiver design for ultrawideband (UWB) communication systems using the channel phase precoding (CPP) technique is proposed in this work. With the CPP-UWB transceiver, we encode data symbols using the reversed order of the channel phase. A simple phase estimation algorithm is presented for the CPP-UWB implementation. Owing to its ability to coherently combine the channel magnitude of every multipath, the CPP-UWB transceiver can achieve a higher data rate by shortening its symbol duration with a tolerable interference. The performance of the CPP-UWB can be further improved using an optimal code length and/or the MMSE receiver to suppress intersymbol interference.
\end{abstract}

Index Terms-Channel phase precoding (CPP), precoder design, precoding, pre-Rake, time-reversal prefilter (TRP), ultrawideband (UWB).

\section{INTRODUCTION}

$\mathbf{O}$ NE distinctive feature of an ultrawideband (UWB) communication system is its remarkable temporal channel resolution. On one hand, the multipath diversity can be potentially exploited by the UWB receiver to combat channel fading. That is, since every path suffers from independent fading, it is less likely that all paths suffer from deep fading simultaneously. On the other hand, the phenomenon that the signal energy is distributed among coefficients of the full channel impulse response (CIR) of long length poses a great challenge for the receiver to collect sufficient signal power for symbol decoding. It was demonstrated in [1] that the receiver demands a large number of correlation operators to achieve this multipath diversity. A receiver of high complexity is, however, not preferred in the UWB system.

The time-reversal prefiltering (TRP) technique, which originates from under water acoustic signal processing, was adopted

Manuscript received January 17, 2006; revised December 6, 2006. This work was supported in part by the Integrated Media Systems Center, a National Science Foundation Engineering Research Center, Cooperative Agreement EEC-9529152, and in part by the National Science Foundation under Contract CCF-0515358. Any opinions, findings, and conclusions or recommendations expressed in this material are those of the authors and do not necessarily reflect those of the National Science Foundation. The associate editor coordinating the review of this manuscript and approving it for publication was Dr. Michael Butler.

Y.-H. Chang, X. Yu, and C.-C. Jay Kuo are with the Ming Hsieh Department of Electrical Engineering, University of Southern California, Los Angeles, CA 90089-2564 USA (e-mail: yuhaocha@usc.edu; xiaoliyu@usc.edu; cckuo@sipi. usc.edu).

S.-H. Tsai is with the Department of Electrical and Control Engineering, National Chiao Tung University, Hsinchu, Taiwan, R.O.C. (e-mail: shanghot@mail.nctu.edu.tw)

Digital Object Identifier 10.1109/TSP.2007.894281 to reduce the UWB receiver complexity by Strohmer et al. [2] When the complete channel information is known at the transmitter, TRP convolves the transmit signal with the time-reversed order of the CIR so that the received signal power can be more concentrated at the receiver. Then, a simple receiver structure with fewer correlation operations can be used. Based on a similar idea, a pre-RAKE scheme was first applied to the CDMA system [3] and later to the UWB channel [4], [5]. Since the TRP transmitter requires the complete channel information, a feedback channel is utilized to send the estimated channel information back to the transmitter. Due to a large number of channel taps is usually encountered, the required bandwidth for the feedback channel is large. Even though TRP can achieve the full multipath diversity, it is somehow difficult to implement in real world UWB systems. To overcome the shortcomings of TRP-based UWB (TRP-UWB) systems, we propose a new UWB transceiver architecture using the channel phase precoding (CPP) technique. The CPP-UWB transmitter utilizes the reversed order of the channel phase as the unique codeword to encode every antipodal data symbol. Note that the phase of each tap of a carrierless UWB channel is either +1 or -1 , i.e., the sign of the corresponding path gain. ${ }^{1}$ If the feedback phase information is accurate, the receiver will get a strong peak signal, which corresponds to a coherent combination of all channel magnitudes. For those off-peak received signals, their amplitudes are much weaker due to incoherent combining. To detect the transmit symbol, the CPP-UWB receiver can apply a decision threshold to the peak received signal. Since the CPP scheme utilizes the phase information only, the receiver can perform phase estimation using a low resolution analog-to-digital converter (ADC), e.g., the 1-bit ADC. As compared with the conventional TRP receiver where a high resolution ADC is demanded to resolve each channel gain, the proposed CPP-UWB receiver can be implemented at a lower cost. Furthermore, each tap's phase can be represented by a single bit so that the transmission of the phase information reduces the feedback channel bandwidth.

A similar idea called "delay tuning" was proposed in [6] to concentrate the received signal power by adjusting the delay and phase of the time-hopped signal properly. However, the delay tuning method and our proposed CPP technique have two major differences. First, the channel model used in [6] assumes that the multipath arrival is random. This assumption complicates algorithms of channel estimation and received signal power focusing. Since the equal-distance, tap-delay UWB channel

\footnotetext{
${ }^{1}$ Even though the phase of a signal $x$ is typically defined in complex baseband as $\arctan \{\operatorname{Im}(x) / \operatorname{Re}(x)\}$, we consider the real baseband signal and view the sign of the signal as its phase information.
} 
model as formulated in [7] was verified by channel measurement results, an efficient channelized codeword is adopted in our work using the tap-delay channel model. Second, by taking advantage of the received power focusing, our system achieves a higher data rate with little intersymbol interference (ISI) penalty at the output of the receiver. In contrast, the delay tuning system adopts a fixed length time-hopping code, which does not leverage the signal power focusing for high data rate communication.

The accuracy of estimated phase information plays an important role in determining the performance of the proposed CPP-UWB system. In this work, we use training symbols for channel phase estimation. Given a fixed number of training symbols, we derive a lower bound on the output signal-to-noise power ratio (SNR) to evaluate the training performance when the data symbol is encoded by the estimated channel phase. The bound becomes tighter as the number of training symbols becomes larger. The use of training symbols degrades the actual data rate for the CPP-UWB system. However, it was also used by TRP to obtain an accurate channel estimation result. With the derived lower bound, a system designer can determine the number of training symbols needed for a given output SNR level. Hence, an excess amount of training can be avoided.

Since CPP-UWB can alleviate the ISI effect by combining channel magnitude gains coherently, it allows the symbol interval to be less than the channel duration to achieve a high data rate while keeping the residual ISI at the peak received signal at a tolerable level. However, as the data rate goes up, the residual ISI becomes more severe and it degrades system performance more than background noise. In this work, we propose two residual ISI mitigation schemes to further improve the system performance of CPP-UWB, namely, the codeword length optimization (CLO) and the MMSE receiver.

The CLO scheme in a CPP-UWB system is motivated by the following observation. Even though a longer codeword (i.e., using more feedback phase information) may result in a higher peak power, the off-peak signal power is also increased, which deteriorates the output signal to interference power ratio (SIR). Consequently, a longer codeword may not necessarily lead to better performance than a shorter one. The main objective of CLO is to adjust the codeword length so that the output SIR is maximized. Furthermore, the use of the optimal code length helps reduce the feedback overhead since the optimal codeword length is usually less or equal to the channel length. Since the CLO problem is highly nonlinear, its closed-form solution is difficult to find. Even though an exhaustive search can be applied for its solution, it is computationally intensive due to the large number of taps of the UWB channel. Here, we propose a fast algorithm to solve the CLO problem to reduce the computational complexity. Even though this fast algorithm does not maximize the output signal to interference plus noise power ratio (SINR), the resulting output SINR only degrades slightly from the optimal one for low input SNR values and converges to the maximum SINR as input SNR increases. When we further decrease the symbol interval to increase the data rate, the CLO scheme alone may not be effective enough in suppressing the residual ISI. Under this case, we can resort to a more sophisticated receiver, e.g., the MMSE receiver, to suppress the interference. This MMSE receiver provides better performance at the cost of additional training symbols to track the MMSE coefficients and higher decoding complexity as compared to the original CPP-UWB receiver.

The rest of the paper is organized as follows. The CPP-UWB system model and its main features are introduced in Section II. The performance of the CPP-UWB system is studied and compared with the existing TRP-UWB scheme [e.g., partial preRAKE (PPR) [4]] in Section III. Then, a simple channel phase estimation scheme is provided and a derived lower bound is used to evaluate the system performance in Section IV. The CLO problem is formulated and a fast search scheme is proposed in Section V. To further suppress the residual interference in higher data rate applications, the use of the MMSE receiver is discussed in Section VI. The issue to maintain the power spectral mask demanded by the U.S. Federal Communications Commission (FCC) [8] is discussed in Section VII, where the output power spectral density (PSD) of the transmitted signal in the CPP-UWB system is derived. Computer simulations are conducted to collaborate the CPP-UWB system in Section VIII. Finally, some concluding remarks are given in Section IX.

\section{SySTEM MODEL AND FEATURES}

\section{A. System Model}

We consider a baseband UWB system that can occupy a wide frequency band, for example, from near DC to several GHz, by employing a proper waveform. The block diagram of the proposed system is given in Fig. 1, where the carrierless, tap-delayed line channel model from Chao and Scholtz [9] is adopted. Thus, the CIR can be written as

$$
h(t)=\sum_{i=0}^{L-1} h_{i} \delta(t-i \Delta)=\sum_{i=0}^{L-1} p_{i} \alpha_{i} \delta(t-i \Delta)
$$

where $\delta(\cdot)$ is the Dirac delta function, $h_{i}=p_{i} \alpha_{i}, L$ is the total number of paths, $\Delta$ is the multipath resolution that is assumed to be the same as the time domain pulse width, $p_{i} \in\{+1,-1\}$ with an equal probability is the phase of the $i$ th path, and $\alpha_{i}$ is the corresponding magnitude which is modeled as an independent Rayleigh random variable with probability density function (PDF) $f_{\alpha_{i}}(x)=\left(x / \sigma_{i}^{2}\right) e^{-x^{2} / 2 \sigma_{i}^{2}}$. The average power of $\alpha_{i}$, which is equal to $2 \sigma_{i}^{2}$, decays exponentially with index $i$, i.e., $E\left\{\alpha_{i}^{2}\right\}=2 \sigma_{i}^{2}=\Omega \gamma^{i}$, where $\Omega$ is the average power of $\alpha_{0}$ and $\gamma \equiv e^{-\Delta / \Gamma}$, and where $\Gamma>\Delta$ is the decay time constant. The time constants of four different UWB channel models [i.e., channel model 1 to 4 (CM 1-4) in [10]] can be found in [9]. Furthermore, we choose $L=\Gamma \eta / \Delta$ to be an integer, where parameter $\eta$ controls the effective length of the channel. In other words, we ignore those taps whose power ratio with respect to the first tap is less or equal to $\gamma^{L}=e^{-\eta}$. It is worthwhile to point out that the equal distance, TDL channel model adopted here is suitable for the dense multipath environment, such as an indoor channel. Since the time delay between different paths could be very small, it is straightforward to treat all propagation paths within one regular time grid as one effective channel tap [7], [11].

As shown in Fig. 1, the receiver first estimates the carrierless channel phase information, which is either +1 or -1 . Then, 


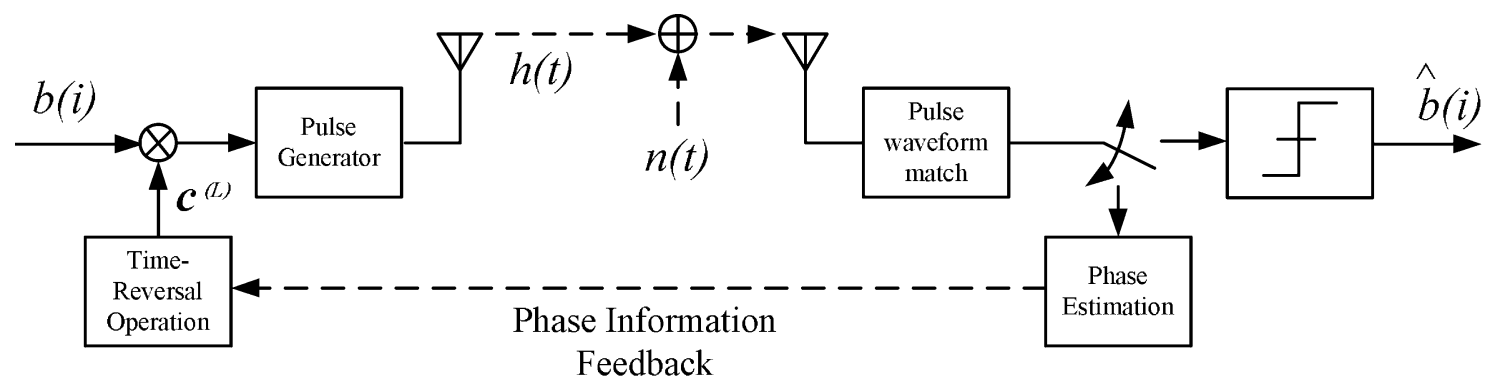

Fig. 1. Block diagram of the CPP-UWB system.

the estimated channel phase information $\hat{\mathbf{p}}$ is sent back to the transmitter as a channelized codeword $\mathbf{c}^{(L)}$, which is time-reversed, unit-power version of $\hat{\mathbf{p}}$. Specifically, $\mathbf{c}^{(L)}$ is given by $\mathbf{c}^{(L)}=\left[c_{0}^{(L)}, \ldots, c_{L-1}^{(L)}\right]^{T}=(1 / \sqrt{L})\left[\hat{p}_{L-1}, \ldots, \hat{p}_{0}\right]^{T}$, where $c_{i}^{(L)}$ is the $i$ th component of the $L$-chip long codeword $\mathbf{c}^{(L)}$ and superscript $T$ represents the transpose.

Afterward, the transmitter encodes each data symbol, $b(i)$, by the channelized codeword $\mathbf{c}^{(L)}$, and then modulates the UWB pulse waveform $w_{\boldsymbol{s}}(t)$ onto each chip. Consequently, the transmit signal can be expressed as

$$
x_{s}(t)=\sum_{i=-\infty}^{\infty} b(i) \sum_{j=0}^{L-1} c_{j}^{(L)} w_{s}\left(t-j \Delta-i T_{s}\right)
$$

where $b(i) \in\{+1,-1\}$ is the $i$ th bipolar data signal, $w_{s}(t)$ is the transmit pulse waveform, and $T_{s} \equiv M \Delta$ is the symbol interval which is assumed to be an integer multiple of the pulse width. Although a more complicated signaling scheme can be applied to $b(i)$, e.g., $m$-level pulse amplitude modulation (PAM) for $m>2$, an additional effort for the amplitude estimation is required. It is not considered in this work.

After the matching and sampling of the received pulse waveform, which is passed through the channel model in (1), the discrete received signal for the $i$ th data symbol $b(i)$ can be written as

$$
\begin{aligned}
\mathbf{r}^{(L)}(i) & =\left[r_{0}^{(L)}(i), \ldots, r_{2 L-2}^{(L)}(i)\right]^{T} \\
& =\mathbf{H}^{(L)} \mathbf{c}^{(L)} b(i)+\mathbf{I}^{(L)}(i)+\mathbf{n}^{(L)}(i)
\end{aligned}
$$

where $\mathbf{H}^{(L)}$ is a $(2 L-1) \times L$ Toeplitz matrix whose first column contains $\mathbf{h}=\left[h_{0}, h_{1}, \ldots, h_{L-1}\right]^{T}$ from the first to the $L$ th elements and zeros elsewhere, $\mathbf{I}^{(L)}(i)=\left[I_{0}^{(L)}(i), \ldots, I_{2 L-2}^{(L)}(i)\right]^{T}$ is the interference vector that contains ISI, and $\mathbf{n}^{(L)}(i)=$ $\left[n_{0}^{(L)}(i), \ldots, n_{2 L-2}^{(L)}(i)\right]^{T}$ is the zero mean, additive white Gaussian noise (AWGN) vector with covariance matrix equal to $\left(N_{o} / 2\right) \mathbf{I}_{2 L-1}$, and where $\mathbf{I}_{2 L-1}$ is the $(2 L-1) \times(2 L-1)$ identity matrix.

Let $\mathbf{h}^{(L)}=\mathbf{H}^{(L)} \mathbf{c}^{(L)}=\left[h_{0}^{(L)}, \ldots, h_{2 L-2}^{(L)}\right]^{T}$ [see (4), shown at the bottom of the page]. When the phase estimation is correct, i.e., $\hat{\mathbf{p}}=\mathbf{p}$, the peak value of $\mathbf{h}^{(L)}$ is given by $\max _{j} h_{j}^{(L)}=$ $\sum_{i=0}^{L-1}\left(\alpha_{i} / \sqrt{L}\right)$ at $j=L-1$ since all channel taps are coherently combined. This is referred to as "signal concentration" in the sequel. To detect transmit symbol $b(i)$, we simply apply the decision threshold to element $r_{L-1}^{(L)}(i)$ in (3), i.e., $\hat{b}(i)=$ $\operatorname{sign}\left\{r_{L-1}^{(L)}(i)\right\}$. Note that when symbol interval $T_{s}$ is greater or equal to the channel response length $L \Delta$, the peak signal used for decoding contains no ISI so that the receiver output is ISI-free. However, owing to the signal concentration effect at the receiver, we can increase the data rate by shortening the symbol interval such that $T_{s}<L \Delta$ by allowing a small ISI penalty at the receiver.

Two ISI mitigation schemes will be presented in Sections V and VI to improve the system performance for the high data rate case. Furthermore, the output SNR can be enhanced by applying the maximum ratio combining (MRC) to both peak and off-peak received signals corresponding to the same transmit symbol. Since MRC demands the amplitude information for the components to be combined, additional training symbols are required for the magnitude estimation and the decoding complexity will increase accordingly. The MRC scheme will not be discussed in this work due to space limitation.

\section{B. Features of CPP-UWB System}

Several interesting features of the proposed CPP-UWB transceiver are described below.

Low Hardware Complexity and Feedback Bandwidth: To provide the channel information to the TRP transmitter, the TRP receiver first identifies the channel response with a high resolution ADC that represents each channel tap with multiple bits (e.g., a 10-bit ADC is utilized in [12]) and then passes those estimated taps back to the transmitter. Since a large number of channel taps are usually observed in the UWB system, the required bandwidth for the feedback channel is large. In contrast, the transmitter of the proposed CPP-UWB system requires the channel phase information only. A low-cost 1-bit ADC can be deployed at the receiver to determine the phase of each tap, and the feedback overhead of the complete phase

$$
h_{i}^{(L)}= \begin{cases}\frac{1}{\sqrt{L}} \sum_{j=0}^{i} \hat{p}_{L-1+j-i} p_{j} \alpha_{j}, & 0 \leq i \leq L-1 \\ \frac{1}{\sqrt{L}} \sum_{j=0}^{2 L-2-i} p_{i+j-L+1} \hat{p}_{j} \alpha_{i+j-L+1}, & L \leq i \leq 2 L-2\end{cases}
$$


information is significantly lower than that of TRP since each phase component can be described by only one bit.

High Data Transmission Rate: If the returned phase information is correct, there will be a strong peak signal at the receiver because of coherent combination of all multipath magnitudes. For a fixed symbol interval, power concentration in the received signal makes possible ISI from neighboring symbols less harmful. For a given noise level at the receiver, we may shorten the symbol interval to achieve a higher data rate due to lower ISI at the corresponding peak received signal. This can be expressed mathematically below. When we encode symbol $b(i)$ with $\mathbf{c}^{(L)}$ for transmission, the received signal of $b(i)$ will spread among $2 L-1$ received samples. Since symbol interval $T_{s}(\equiv M \Delta)$ is now less than $L \Delta, L_{0}(\equiv\lfloor(L-1) / M\rfloor)$ preceding and $L_{0}$ succeeding peak signals are interfered by off-peak signals of symbol $b(i)$, where $\lfloor x\rfloor$ is the floor function of $x$. To state this alternatively, $L_{0}$ preceding and $L_{0}$ succeeding data symbols of $b(i)$ will contribute their off-peak signals to the peak received signal of $b(i)$. Given a maximal noise level $\varepsilon$, the fastest data rate, i.e., the shortest allowable symbol interval $M_{\min }$, can be determined by

$$
\begin{aligned}
M_{\min }= & \arg \min _{M} \sum_{j=1}^{L_{0}} E\left\{\left(h_{L+j M-1}^{(L)} b(i-j)\right)^{2}\right. \\
& \left.+\left(h_{L-j M-1}^{(L)} b(i+j)\right)^{2}\right\} \\
= & \arg \min _{M} \sum_{j=1}^{L_{0}} E\left\{\left(h_{L+j M-1}^{(L)}\right)^{2}\right. \\
& \left.+\left(h_{L-j M-1}^{(L)}\right)^{2}\right\} \leq \varepsilon-\frac{N_{o}}{2}
\end{aligned}
$$

where $E\left\{\left(h_{L+j M-1}^{(L)}\right)^{2}\right\}$ and $E\left\{\left(h_{L-j M-1}^{(L)}\right)^{2}\right\}$ are the interference terms contributed by preceding and succeeding symbols of current symbol $b(i)$, respectively.

Secure Data Transmission: According to the experimental result in [13], the spatial correlation between two UWB channels is less than 0.1 if two receivers are separated by more than 10 in. Thus, the channel of each transceiver pair can be viewed as being independent. The channelized codeword that is random in nature can be used to encode every transmit symbol, which makes eavesdropping more difficult. Even though the third party may acquire the codeword information during the feedback stage of the phase information, the attacker has to overcome a serious ISI effect in order to decode the received data correctly in other locations. Hence, the CPP-UWB system can achieve higher security in transmission by the nature of its design. We should point out that TRP can achieve high-rate and secure data transmission as well. Since more channel information is utilized at the TRP transmitter, its performance is better than CPP-UWB in secure and high-rate data transmission yet at the expense of higher feedback overhead and a more expensive $\mathrm{ADC}$ at the TRP receiver.

\section{OUTPUT SNR AND SINR ANALYSIS}

In this section, we will analyze the performance of the CPP-UWB system in terms of output SNR and SINR for different temporal resolutions, with $T_{s} \geq L \Delta$ and $T_{s} \leq L \Delta$, respectively. The returned phase information is assumed to be perfect, i.e., $\hat{\mathbf{p}}=\mathbf{p}$, in our following analysis.

\section{A. Output SNR Analysis for Low Data Rate Case}

When the data rate is low, symbol intervals are longer, i.e., $T_{s}>L \Delta$. In this case, the received peak signal is free from ISI. Then, the output SNR using $L$-chip long codeword can be calculated as

$$
\bar{\nu}^{(L)}=\frac{E\left\{\left(h_{L-1}^{(L)} b(i)\right)^{2}\right\}}{E\left\{\left(n_{L-1}^{(L)}(i)\right)^{2}\right\}}=\frac{\bar{P}_{h_{L-1}^{(L)}}}{N_{0} / 2}
$$

where $\bar{P}_{h_{L-1}^{(L)}}$ is defined as

$$
\begin{aligned}
\bar{P}_{h_{L-1}^{(L)}} & \equiv E\left\{\left(h_{L-1}^{(L)} b(i)\right)^{2}\right\} \\
& =E\left\{\left(\frac{1}{\sqrt{L}} \sum_{j=0}^{L-1} \alpha_{j}\right)^{2}\right\} \\
& =\frac{1}{L} E\left\{\sum_{j=0}^{L-1} \alpha_{j}^{2}+\sum_{l, m=0 ; l \neq m}^{L-1} \alpha_{l} \alpha_{m}\right\}
\end{aligned}
$$

is the average peak power. Since $\alpha_{l}$ and $\alpha_{m}$ are independent, and the first and the second moments of the Rayleigh random variable $\alpha_{j}$ equal $\left(\sqrt{\pi \Omega \gamma^{j}} / 2\right)$ and $\Omega \gamma^{j}$, respectively, we can further simplify (6) as

$$
\begin{aligned}
\bar{P}_{h_{L-1}^{(L)}} & =\frac{\Omega\left(1-\gamma^{L}\right)}{L(1-\gamma)}+\frac{\Omega \pi}{4 L}\left(\sum_{l, m=0 ; l \neq m}^{L-1} \gamma^{(l+m) / 2}\right) \\
& =\frac{\Omega\left(1-\gamma^{L}\right)}{L(1-\gamma)}+\frac{\Omega \pi}{4 L}\left(\left(\sum_{l=0}^{L-1} \gamma^{l / 2}\right)^{2}-\sum_{l=0}^{L-1} \gamma^{l}\right) \\
& =\left(1-\frac{\pi}{4}\right) \frac{\Omega\left(1-\gamma^{L}\right)}{L(1-\gamma)}+\frac{\Omega \pi}{4 L}\left(\frac{1-\gamma^{L / 2}}{1-\gamma^{1 / 2}}\right)^{2} .
\end{aligned}
$$

Let $\varsigma$ be the ratio between the second and the first terms at the right-hand side of (7), which can be bounded from below by

$$
\varsigma=\frac{\frac{\Omega \pi}{4 L}\left(\frac{1-\gamma^{L / 2}}{1-\gamma^{1 / 2}}\right)^{2}}{\left(1-\frac{\pi}{4}\right) \frac{\Omega\left(1-\gamma^{L}\right)}{L(1-\gamma)}} \geq \frac{\pi}{4-\pi} \frac{2 \Gamma}{\Delta}\left(\frac{1-e^{-\eta / 2}}{1+e^{-\eta / 2}}\right) .
$$

It will be shown in Example 1 of Section VIII that, for a typical value of $\eta$ (say, $\eta=5.85$ ), we have

$$
\varsigma \geq 50 \Longleftrightarrow \Gamma / \Delta \geq 7.6 \text {. }
$$

Since $\Gamma \gg \Delta, \varsigma>50$ and $\bar{P}_{h_{L-1}^{(L)}}$ is dominated by the second term at the right-hand side of (7), we can get the following approximation:

$$
\bar{P}_{h_{L-1}^{(L)}} \approx \frac{\Omega \pi}{4 L}\left(\frac{1-\gamma^{L / 2}}{1-\gamma^{1 / 2}}\right)^{2}
$$


Please note that the value of $\Omega$ is set as

$$
\Omega=\frac{1-\gamma}{1-\gamma^{L}}
$$

so that the overall power spread in the UWB channel is unity. By substituting (10) into (9), $\bar{P}_{h_{L-1}^{(L)}}$ is approximated by

$$
\begin{aligned}
\bar{P}_{h_{L-1}^{(L)}} & \approx \frac{\pi}{4 L} \frac{\left(1+\gamma^{1 / 2}\right)\left(1-\gamma^{L / 2}\right)}{\left(1+\gamma^{L / 2}\right)\left(1-\gamma^{1 / 2}\right)} \\
& =\frac{\pi \Delta}{4 \Gamma \eta} \frac{\left(1-e^{-\eta / 2}\right)\left(1+e^{-\Delta / 2 \Gamma}\right)}{\left(1+e^{-\eta / 2}\right)\left(1-e^{-\Delta / 2 \Gamma}\right)}
\end{aligned}
$$

where $L$ is replaced by $\Gamma \eta / \Delta$ to get the last equation. Furthermore, since $\Delta / 2 \Gamma \ll 1$, we have

$$
1+e^{-\Delta / 2 \Gamma} \approx 2 \text { and } 1-e^{-\Delta / 2 \Gamma} \approx \Delta / 2 \Gamma
$$

by ignoring higher order terms in the Taylor series expansion of $e^{-\Delta / 2 \Gamma}$. Then, with (5), (11), (12), and some manipulations, we have

$$
\bar{\nu}^{(L)} \approx \frac{2 \pi\left(1-e^{-\eta / 2}\right)}{N_{0} \eta\left(1+e^{-\eta / 2}\right)} .
$$

As shown in (13), the output SNR depends only on variable $\eta$ that controls the effective length of the UWB channel in our model.

Since the CPP transmitter uses only the channel phase information to encode its transmit symbol, the CPP receiver acquires less signal power as compared to the ideal TRP method. Let $\bar{\nu}_{\text {TRP }}$ be the output SNR at the peak received signal of the TRP system. It can be calculated by

$$
\bar{\nu}_{\mathrm{TRP}}=\frac{E\left\{\sum_{i=0}^{L-1}\left(p_{i} \alpha_{i}\right)^{2}\right\}}{N_{0} / 2}=\frac{2 \Omega\left(1-e^{-\eta}\right)}{N_{0}\left(1-e^{-\Delta / \Gamma}\right)}=\frac{2}{N_{0}} .
$$

This result is not surprising since TRP achieves full multipath diversity at the receiver end and the channel power has been normalized to one. Let $\varphi$ be the SNR degradation factor defined by

$$
\varphi=\frac{\bar{\nu}^{(L)}}{\bar{\nu}_{\mathrm{TRP}}} .
$$

Based on (13)-(15), we can show that

$$
\varphi \approx \frac{\pi\left(1-e^{-\eta / 2}\right)}{\eta\left(1+e^{-\eta / 2}\right)}
$$

Both the output SNR in (13) and the degradation factor given above depend on variable $\eta$, which is fixed in our channel model. For a large value of $\eta$, the peak power of CPP-UWB may decrease to result in the reduction of $\bar{\nu}^{(L)}$ and $\varphi$. This can be explained by the fact that, when there are more trivial taps in the channel, combining all channel magnitudes may fail to improve the peak signal power since each emitted pulse is normalized by $\sqrt{L}$. Several approximations, such as (9) and (12), are used to get a clean result of (16). We will show the validity of these approximations in Section VIII.

\section{B. Output SINR Analysis for High Data Rate Case}

When the data rate is high, symbol intervals are shorter. In particular, if $T_{s}<L \Delta$, the received signal $r_{L-1}^{(L)}(i)$ contains not only the desired signal contributed by $b(i)$ (i.e., $h_{L-1}^{(L)} b(i)$ ) but also the interference contributed by its neighboring symbols. The corresponding SINR can be written as the equation shown at the bottom of the page, where $L_{0}$ was defined in Section II. We first consider the postcursor ISI, i.e., $E\left\{\left(h_{L-1-j M}^{(L)} b(i+j)\right)^{2}\right\}$, $1 \leq j \leq L_{0}$. Let $\eta_{1}=\Delta(L-j M) / \Gamma$ be a real number. The interference power from $b(i+j)$ in $r_{L-1}^{(L)}(i)$ can be calculated as

$$
\begin{aligned}
\bar{P}_{h_{L-1-j M}^{(L)}} & =E\left\{\left(h_{L-1-j M}^{(L)} b(i+j)\right)^{2}\right\} \\
& =\frac{1}{L} E\left\{\left(\sum_{n=0}^{L-1-j M} p_{n+j M} p_{n} \alpha_{n}\right)^{2}\right\} .
\end{aligned}
$$

Since $p_{i}, i=0, \ldots, L-1$, are independent zero-mean random variables, we know that the expected value of the cross term in (17) is zero. Therefore, $\bar{P}_{h_{L-1-j M}^{(L)}}$ can be simplified as

$$
\begin{aligned}
\bar{P}_{h_{L-1-j M}^{(L)}} & =\frac{1}{L} E\left\{\sum_{n=0}^{L-1-j M} \alpha_{n}^{2}\right\} \\
& =\frac{1}{L} \sum_{n=0}^{L-1-j M} \Omega \gamma^{n}=\frac{\Omega}{L} \frac{1-\gamma^{L-j M}}{1-\gamma} .
\end{aligned}
$$

By substituting $\gamma=e^{-\Delta / \Gamma}$ and $L-j M=\Gamma \eta_{1} / \Delta$ into (18), we have

$$
\bar{P}_{h_{L-1-j M}^{(L)}}=\frac{\Omega}{L} \frac{1-e^{-\eta_{1}}}{1-e^{-\Delta / \Gamma}} .
$$

$$
\bar{\nu}^{(L)}=\frac{E\left\{\left(h_{L-1}^{(L)} b(i)\right)^{2}\right\}}{E\left\{\left(n_{L-1}^{(L)}(i)\right)^{2}\right\}+\sum_{j=1}^{L_{0}} E\left\{\left(h_{L-1+j M}^{(L)} b(i-j)\right)^{2}\right\}+\sum_{j=1}^{L_{0}} E\left\{\left(h_{L-1-j M}^{(L)} b(i+j)\right)^{2}\right\}}
$$


Thus, with (9) and (19), the power ratio between $\bar{P}_{h_{L-1}^{(L)}}$ and $\bar{P}_{h_{L-j M-1}^{(L)}}$ can be found as

$$
\begin{aligned}
\frac{\bar{P}_{h_{L-1}^{(L)}}}{\bar{P}_{h_{L-j M-1}^{(L)}}} \approx \frac{\frac{\Omega \pi}{4 L}\left(\frac{1-e^{-\eta / 2}}{1-e^{-\Delta / 2 \Gamma}}\right)^{2}}{\frac{\Omega}{L} \frac{1-e^{-\eta} 1}{1-e^{-\Delta / \Gamma}}} \approx \frac{\mathcal{C}}{\Delta} \\
\mathcal{C}=\frac{\pi \Gamma\left(1-e^{-\eta / 2}\right)^{2}}{1-e^{-\eta_{1}}}
\end{aligned}
$$

where $\mathcal{C}$ is independent of $\Delta$. Thus, the decrease of $\Delta$ boosts the power ratio between $\bar{P}_{h_{L-1}^{(L)}}$ and $\bar{P}_{h_{L-j M-1}^{(L)}}$, which is the interference power provided by $b(i+j), 1 \leq j \leq L_{0}$. It is easy to generalize the above result to the precursor ISI analysis, which is contributed by $b(i-j), 1 \leq j \leq L_{0}$. We conclude that the improvement of temporal resolution provides a higher output SINR when the input SNR is fixed. Please note that the feedback overhead is also increased due to more multipath components are resolved.

\section{Comparison Between TRP and CPP Schemes}

Due to the very large tap number in the UWB channel, the feedback of complete channel information as demanded by an ideal TRP scheme is actually not practical. Two channel-tap selection schemes; namely, selective pre-RAKE (SPR) and partial pre-RAKE (PPR) [4], [14], were proposed to reduce the feedback overhead. It was demonstrated in [14] that the PPR system that delivers the first several channel taps to the transmitter can achieve the maximum average peak power if a fixed number of consecutive taps are available from the feedback channel. Both CPP-UWB and PPR-UWB provide incomplete channel information to the transmitter; namely, the channel phase in CPP and the first several channel taps in PPR. It is interesting to know the number of channel taps required for the PPR transmitter to achieve the same peak received power when a $l$-chip (with $l \leq L$ ) long codeword is used in the CPP transmitter.

With the channel model in Section II, the power concentration by the PPR scheme using the first $\bar{L}$ taps was derived in [14] as

$$
\bar{P}_{\mathrm{PPR}}(\bar{L})=\Omega \frac{1-\gamma^{\bar{L}}}{1-\gamma} .
$$

When an $l$-chip long codeword in the CPP scheme achieves the same power concentration at the peak of PPR using the first $\bar{L}$ taps, we can set $P_{\mathrm{PPR}}(\bar{L})=\bar{P}_{h_{l-1}^{(l)}}\left(\left.\equiv \bar{P}_{h_{L-1}^{(L)}}\right|_{L=l}\right)$, and get $\gamma^{\bar{L}}=1-\rho$, where $\rho=(1 / l)(1-(\pi / 4))\left(1-\gamma^{l}\right)+(\pi / 4 l)$ $\left(\left(1-\gamma^{l / 2}\right) /\left(1-\gamma^{1 / 2}\right)\right)^{2}(1-\gamma)$. Since $\bar{L}$ is a positive number, we may select

$$
\bar{L}=\left\lceil-\frac{\Gamma}{\Delta} \ln (1-\rho)\right\rceil
$$

where $\lceil x\rceil$ is the ceiling function of $x$. We will verify the result derived above by computer simulation in Section VIII. We compare the required feedback overhead of PPR and CPP to achieve roughly the same output signal power. Thus, given a fixed amount of feedback information, their bit-error-rate (BER) performance should be close to each other when the peak received signal is ISI-free.
It is worthwhile to mention that (22) may not guarantee the same output SINR for both systems when the symbol interval is less than the channel response length. However, since the output SINR is a highly nonlinear function of the codeword length, a closed-form solution to the feedback overhead is difficult to obtain. In fact, as suggested by simulation results in Section VIII, the BER gap between these two systems is small when (22) is applied. Thus, our analysis is still valid even when ISI occurs.

\section{Phase Estimation And Performance Analysis WITH ESTIMATED PHASE INFORMATION}

Accurate phase estimation is critical to the performance of the CPP-based transceiver. In this section, we first present a simple yet practical phase estimation algorithm based on training symbols. Then, based on the estimated phase with $N$ training symbols, a lower bound on the output SNR using the $L$-chip long codeword will be derived to evaluate the system performance, where the output SNR can be expressed as

$$
\bar{\nu}^{(L)}=\frac{E\left\{\left(h_{L-1}^{(L)} b(i)\right)^{2}\right\}}{E\left\{\left(n_{L-1}^{(L)}(i)\right)^{2}\right\}}=\frac{2 E\left\{\left(h_{L-1}^{(L)}\right)^{2}\right\}}{N_{0}} .
$$

The performance lower bound is provided for the ISI-free data transmission. However, a similar idea can be easily used to derive the lower bound on the output SINR when $T_{s}<L \Delta$.

\section{A. Channel Phase Estimation Algorithm}

The following channel phase estimation algorithm is adopted in the proposed CPP system.

1) In the initialization stage, the transmitter emits $N$ channel sounding pulses, $b_{t}(0), \ldots, b_{t}(N-1)$, of a low duty cycle so that the received signals of different transmit symbols do not overlap with each other.

2) After the received signal is synchronized, the receiver matches the received pulse waveform and takes sample at every $\Delta$ time instance to digitalize received data.

3) All $N$ discrete received signals are demodulated and then averaged to reduce the noise perturbation before the phase estimation task.

4) The estimated channel phase is given by

$$
\begin{aligned}
\hat{\mathbf{p}} & =\operatorname{sign}\left\{\frac{1}{N} \sum_{l=0}^{N-1} b_{t}(l) \mathbf{r}_{t}(l)\right\} \\
& =\operatorname{sign}\left\{\frac{1}{N} \sum_{l=0}^{N-1} b_{t}(l)\left(\mathbf{h} b_{t}(l)+\mathbf{n}_{t}(l)\right)\right\} \\
& =\operatorname{sign}\left\{\mathbf{h}+\mathbf{n}_{t}^{(N)}\right\}
\end{aligned}
$$

where $\mathbf{r}_{t}(l)$ is the $l$ th received signal vector, $\mathbf{n}_{t}(l)$ is the $l$ th AWGN noise vector with zero-mean and covariance matrix $\left(N_{0} / 2\right) \mathbf{I}_{L}$, and

$$
\begin{aligned}
\mathbf{n}_{t}^{(N)} & \equiv \frac{1}{N} \sum_{l=0}^{N-1} \mathbf{n}_{t}(l) b_{t}(l) \\
& =\left[n_{t, 0}^{(N)}, \ldots, n_{t, L-1}^{(N)}\right]^{T}
\end{aligned}
$$


which is a zero-mean Gaussian random vector whose elements are independent, identically distributed (i.i.d.) Gaussian random variables with variance equal to $N_{0} /(2 N)$.

\section{B. Performance Analysis With Estimated Phase}

In the following proposition, we give a performance bound on the CPP system using the estimated channel phase information to encode its data symbols.

Proposition 1: Consider a CPP system that uses $N$ training symbols for channel phase estimation. Then, the output SNR as given in (23) satisfies

$$
\begin{aligned}
\bar{\nu}^{(L)} & \geq \frac{2}{L N_{0}}\left\{\Omega \frac{1-\gamma^{L}}{1-\gamma}\right. \\
+ & \sum_{i, j=0 ; i \neq j}^{L-1}\left[\frac{\sqrt{\pi \Omega}}{2} \gamma^{i / 2}-\frac{\sqrt{2 \pi}}{\Omega \gamma^{i}}\left(\frac{\Omega N_{0} \gamma^{i}}{2 N_{0}+2 N \Omega \gamma^{i}}\right)^{3 / 2}\right] \\
& \left.\cdot\left[\frac{\sqrt{\pi \Omega}}{2} \gamma^{j / 2}-\frac{\sqrt{2 \pi}}{\Omega \gamma^{j}}\left(\frac{\Omega N_{0} \gamma^{j}}{2 N_{0}+2 N \Omega \gamma^{j}}\right)^{3 / 2}\right]\right\} .
\end{aligned}
$$

Proof: Let $\rho_{i}$ be the product of the $i$ th phase component $p_{i}$ and its estimate $\hat{p}_{i}$, i.e., $\rho_{i} \equiv p_{i} \hat{p}_{i}$. $\rho_{i}$ has two possible values, i.e., 1 or -1 , which reveals whether the $i$ th estimate is correct. The average desired signal power concentrated at the peak is given by

$$
\begin{aligned}
E & \left\{\left(\frac{1}{\sqrt{L}} \sum_{l=0}^{L-1} \rho_{l} \alpha_{l} b(i)\right)^{2}\right\} \\
& =E\left\{\left(\frac{1}{\sqrt{L}} \sum_{l=0}^{L-1} \rho_{l} \alpha_{l}\right)^{2}\right\} \\
& =\frac{1}{L} E\left\{\sum_{l=0}^{L-1} \alpha_{l}^{2}\right\}+\frac{1}{L} E\left\{\sum_{l, m=0 ; l \neq m}^{L-1} \rho_{l} \alpha_{l} \rho_{m} \alpha_{m}\right\} \\
& =\frac{1}{L} \sum_{l=0}^{L-1} \Omega \gamma^{l}+\frac{1}{L} \sum_{l, m=0 ; l \neq m}^{L-1} E\left\{\rho_{l} \alpha_{l}\right\} E\left\{\rho_{m} \alpha_{m}\right\} .
\end{aligned}
$$

When the phase is estimated by (24), the conditional error probability on the $i$ th phase is

$$
\begin{aligned}
& \operatorname{Pr}\left\{\rho_{i}=-1 \mid \alpha_{i}\right\} \\
& \quad=\operatorname{Pr}\left\{\hat{p}_{i} \neq p_{i} \mid \alpha_{i}\right\} \\
& =\operatorname{Pr}\left\{p_{i} \alpha_{i}+n_{t, i}^{(N)}<0 \mid p_{i}=1 ; \alpha_{i}\right\} \operatorname{Pr}\left\{p_{i}=1\right\} \\
& \quad+\operatorname{Pr}\left\{p_{i} \alpha_{i}+n_{t, i}^{(N)}>0 \mid p_{i}=-1 ; \alpha_{i}\right\} \operatorname{Pr}\left\{p_{i}=-1\right\} \\
& =Q\left(\sqrt{2 N \alpha_{i}^{2} / N_{0}}\right)
\end{aligned}
$$

where $n_{t, i}^{(N)}$ is the $i$ th element of $\mathbf{n}_{t}^{(N)}$ and $Q(x) \equiv$ $\int_{x}^{\infty}(1 / \sqrt{2 \pi}) e^{-t^{2} / 2} d t$. Therefore, using the result in (29), we can simplify $E\left\{\rho_{i} \alpha_{i} \mid \alpha_{i}\right\}$ as

$$
\begin{aligned}
E\left\{\rho_{i} \alpha_{i} \mid \alpha_{i}\right\} & \\
= & \left.\rho_{i} \alpha_{i}\right|_{\rho_{i}=1} \cdot \operatorname{Pr}\left\{\rho_{i}=1 \mid \alpha_{i}\right\} \\
& +\left.\rho_{i} \alpha_{i}\right|_{\rho_{i}=-1} \cdot \operatorname{Pr}\left\{\rho_{i}=-1 \mid \alpha_{i}\right\} \\
= & \alpha_{i} \cdot\left(1-\operatorname{Pr}\left\{\rho_{i}=-1 \mid \alpha_{i}\right\}\right)-\alpha_{i} \cdot \operatorname{Pr}\left\{\rho_{i}=-1 \mid \alpha_{i}\right\} \\
= & \alpha_{i}-2 Q\left(\sqrt{2 N \alpha_{i}^{2} / N_{0}}\right) \alpha_{i} .
\end{aligned}
$$

Thus, by taking the expectation operator with respect to variable $\alpha_{i}$ at both sides of the above equation, we get

$$
\begin{aligned}
E\left\{\rho_{i} \alpha_{i}\right\} & =E_{\alpha_{i}}\left\{E\left\{\rho_{i} \alpha_{i} \mid \alpha_{i}\right\}\right\} \\
& =E\left\{\alpha_{i}\right\}-2 \int_{0}^{\infty} Q\left(\sqrt{2 N x^{2} / N_{0}}\right) x f_{\alpha_{i}}(x) d x \\
& \geq E\left\{\alpha_{i}\right\}-\int_{0}^{\infty} e^{-N x^{2} / N_{0}} x f_{\alpha_{i}}(x) d x
\end{aligned}
$$

where the upper bound on $Q(x)$ given in [15], i.e., $Q(x) \leq$ $(1 / 2) e^{-x^{2} / 2}$, is applied to get the inequality in (31). After some manipulations, (31) becomes

$$
E\left\{\rho_{i} \alpha_{i}\right\} \geq \frac{\sqrt{\pi \Omega}}{2} \gamma^{i / 2}-\frac{\sqrt{2 \pi}}{\Omega \gamma^{i}}\left(\frac{\Omega N_{0} \gamma^{i}}{2 N_{0}+2 N \Omega \gamma^{i}}\right)^{3 / 2} .
$$

By substituting (32) into (27) and dividing (27) by the noise power $N_{0} / 2$, Proposition 1 is proved.

The above lower bound gives users an idea about the system performance when $N$ training symbols are used. Thus, users can adjust the number of training symbols to meet a specific performance requirement while minimizing the training overhead. It is straightforward to show that the mean-square-error (MSE) of the proposed phase estimation scheme is

$$
E\left\{|\mathbf{p}-\hat{\mathbf{p}}|^{2}\right\}=\sum_{i=0}^{L-1} 4 \cdot E_{\alpha_{i}}\left\{Q\left(\sqrt{2 N \alpha_{i}^{2} / N_{0}}\right)\right\} .
$$

By the similar method used to develop the lower bound on $\bar{\nu}^{(L)}$, we can have the upper bound for $E\left\{|\mathbf{p}-\hat{\mathbf{p}}|^{2}\right\}$ as

$$
E\left\{|\mathbf{p}-\hat{\mathbf{p}}|^{2}\right\} \leq 2 \sum_{i=0}^{L-1} \frac{N_{0}}{N_{0}+\Omega \gamma^{i} N} .
$$

Also, we can show that the conditional mean of $\hat{p}_{i}$ is equal to

$$
E\left\{\hat{p}_{i} \mid p_{i}, \alpha_{i}\right\}=\left(1-Q\left(\sqrt{2 N \alpha_{i}^{2} / N_{0}}\right)\right) p_{i}
$$

which is asymptotically unbiased as $N$ approaches infinity. The unconditional zero mean of $\hat{p}_{i}$ suggests that the proposed simple phase estimation method is unbiased. The above sample average scheme can be used to identify the current channel response for TRP, as well. 


\section{CODEWORD LENGTH OPTIMIZATION (CLO)}

\section{A. Problem Statement}

By exploiting the power concentration property of received signals, we can shorten the symbol interval to achieve a higher data rate without inducing much ISI at the peak value. On the other hand, when the data rate is sufficiently high, the system performance degrades greatly due to strong ISI at the receiver output, which dominates the performance more than background noise. Although we may increase the peak signal power by combining more channel taps with a longer codeword, this long codeword may amplify the off-peak received signals to cause interference to peak signals of neighboring symbols. Hence, for a fixed symbol interval, there exists an optimal codeword length that maximizes the output signal to interference ratio (SIR) at the peak received signal. The knowledge of the optimal codeword length helps reduce the feedback overhead since it is less or equal to the CIR length.

Example 1: Output SNR (or SINR) Versus Codeword Length: We demonstrate the output SIR as a function of the codeword length in this example. The system parameters are $L=240$, $M=40, \Delta=0.5 \mathrm{~ns}$ and $\Gamma=20.5 \mathrm{~ns}$ (CM3). Here, we consider two different codeword lengths: $l=240$ and $l=30$. They correspond to the full length and the partial length cases, respectively. The received signal power with respect to one channel realization is plotted in Fig. 2. In Fig. 2, the star denotes the position of the peak signal of a target symbol while circles denote positions of the peak signal of its neighboring symbols. Furthermore, we also list the values of the peak signal power and its SIR for reference. As shown in this example, although a longer codeword may provide a higher peak power, the output SIR is actually smaller.

In this section, we consider the codeword length optimization (CLO) problem to improve the performance of the CPP scheme with a fixed data rate. For simplicity, the feedback phase information is assumed to be perfect and the channel length is an integer multiple of the symbol interval, i.e., $L=K M$, where $K$ is an positive integer and $M=T_{s} / \Delta$. The latter requirement may not be as restrictive as it appears, since we can either zero-pad or truncate the channel length to satisfy this assumption when the amplitude of the channel tap becomes very small. We will show that the system performance of this altered channel is almost indistinguishable from that of the original channel in Section VIII.
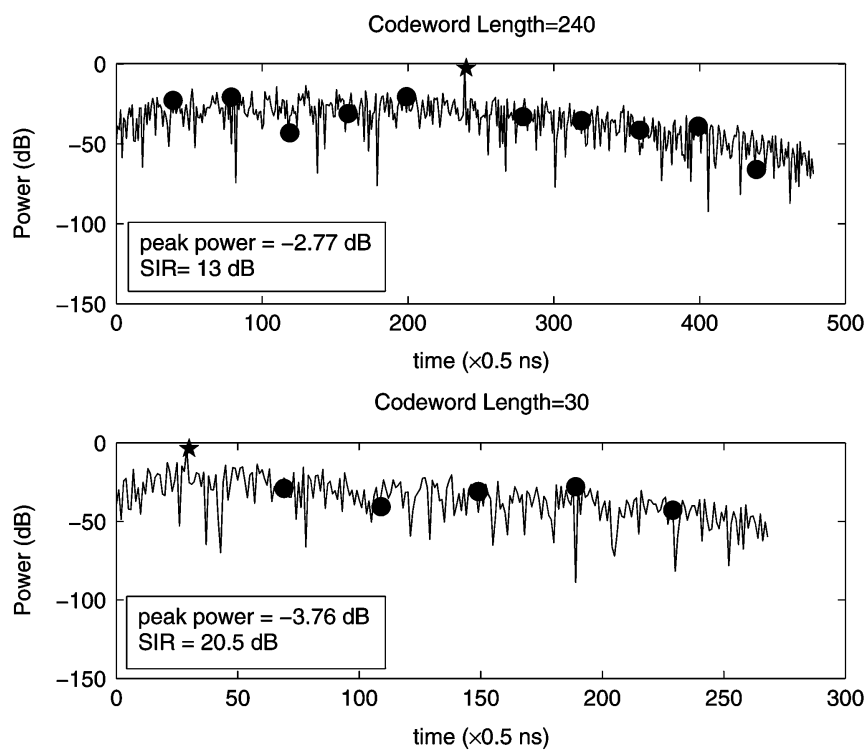

Fig. 2. Received signal power for different codeword lengths, where $\Delta=0.5$ ns, $\Gamma=20.5 \mathrm{~ns}(\mathrm{CM} 3)$, and $L=240$.

Let $l$ be the length of codeword $\mathbf{c}^{(l)}$ where $\mathbf{c}^{(l)}=$ $\left[c_{0}^{(l)}, \ldots, c_{l-1}^{(l)}\right]^{T}=(1 / \sqrt{l})\left[p_{l-1}, \ldots, p_{0}\right]^{T}$. When transmit symbol $b(i)$ is encoded by $\mathbf{c}^{(l)}$, the received signal can be written as

$$
\begin{aligned}
\mathbf{r}^{(l)}(i) & =\left[r_{0}^{(l)}(i), \ldots, r_{L+l-2}^{(l)}(i)\right]^{T} \\
& =\mathbf{H}^{(l)} \mathbf{c}^{(l)} b(i)+\mathbf{I}^{(l)}(i)+\mathbf{n}^{(l)}(i) \\
& =\mathbf{h}^{(l)} b(i)+\mathbf{I}^{(l)}(i)+\mathbf{n}^{(l)}(i)
\end{aligned}
$$

where $\mathbf{H}^{(l)}$ is the $(L+l-1) \times l$ Toeplitz matrix, $\mathbf{I}^{(l)}(i)$ and $\mathbf{n}^{(l)}(i)$ are the interference and AWGN vectors, respectively, $\mathbf{h}^{(l)}=\mathbf{H}^{(l)} \mathbf{c}^{(l)}=\left[h_{0}^{(l)}, \ldots, h_{L+l-1}^{(l)}\right]^{T}$ [see (36), shown at the bottom of the page].

Since the first $l$ channel taps are coherently summed, it is clear that the maximum signal power occurs at $h_{l-1}^{(l)}(i)$ and the average output SIR at $r_{l-1}^{(l)}(i)$ is equal to (37), shown at the bottom of the page. The optimal codeword length $L_{\mathrm{opt}}$ can be found as

$$
L_{\mathrm{opt}}=\arg \max _{0<l \leq L} \bar{\nu}^{(l)}
$$

$$
h_{i}^{(l)}= \begin{cases}\frac{1}{\sqrt{l}} \sum_{j=0}^{i} p_{l-1+j-i} p_{j} \alpha_{j}, & 0 \leq i \leq l-1 \\ \frac{1}{\sqrt{l}} \sum_{j=0}^{l-1} p_{j} p_{j+i-l+1} \alpha_{j+i-l+1}, & l \leq i \leq L-1 \\ \frac{1}{\sqrt{l}} \sum_{j=0}^{l-i+L-2} p_{j} p_{l-1+j} \alpha_{l-1+j}, & L \leq i \leq L+l-2\end{cases}
$$

$$
\bar{\nu}^{(l)}=\frac{E\left\{\left(h_{l-1}^{(l)} b(i)\right)^{2}\right\}}{\sum_{j=1}^{\lfloor(L-1) / M\rfloor} E\left\{\left(h_{l+j M-1}^{(l)} b(i-j)\right)^{2}\right\}+\sum_{j=1}^{L(l-1) / M\rfloor} E\left\{\left(h_{l-j M-1}^{(l)} b(i+j)\right)^{2}\right\}}
$$


where a closed-form solution to $L_{\mathrm{opt}}$ is difficult to get due to the nonlinear nature of the problem. An exhaustive search algorithm that tests all possible values of $l$ is computationally intensive. We will present a fast search algorithm to find the optimal codeword length instead in Section V-B.

Please note that we focus on the CLO problem primarily for the high data rate scenario, i.e., $T_{s} \leq L \Delta$. It is worthwhile to point out that there still exists an optimal code length so that the output SNR is maximized even when the received signal to be decoded is ISI-free. This is due to the fact that the average power of the channel tap decays exponentially with respect to its index and we normalize the output power by dividing codeword $\mathbf{c}^{(l)}$ by $\sqrt{l}$ so that a longer codeword may or may not increase the peak received power. The CLO problem for the low data rate case is, however, not as interesting as that for the high data rate case.

\section{B. Fast Search Algorithm for Optimal Code Length}

By substituting (36) into (37), and after some manipulations, we can express the output SIR at the peak received signal as

$$
\bar{\nu}^{(l)}=\frac{E\left\{\left(\sum_{j=0}^{l-1} \alpha_{j}\right)^{2}\right\}}{\sum_{j=0}^{l-1} I^{(j)}}
$$

where

$$
\begin{aligned}
I^{(j)} \equiv & \sum_{m=1}^{\lfloor j / M\rfloor} E\left\{\left(\alpha_{j-m M} p_{j} p_{j-m M} b(i+m)\right)^{2}\right\} \\
& +\sum_{n=1}^{\lfloor(L-j) / M\rfloor} E\left\{\left(\alpha_{j+n M} p_{j} p_{j+n M} b(i-n)\right)^{2}\right\} \\
= & \sum_{m=1}^{\lfloor j / M\rfloor} E\left\{\alpha_{j-m M}^{2}\right\}+\sum_{n=1}^{\lfloor(L-j) / M\rfloor} E\left\{\alpha_{j+n M}^{2}\right\} \\
= & \sum_{m=1}^{\lfloor j / M\rfloor} \Omega \gamma^{j-m M}+\sum_{n=1}^{\lfloor(L-j) / M\rfloor} \Omega \gamma^{j+n M}
\end{aligned}
$$

is the normalized interference power generated by adding the $j$ th channel tap at the peak and $\sum_{m=1}^{\lfloor j / M\rfloor} \Omega \gamma^{j-m M}$ and $\sum_{n=1}^{\lfloor(L-j) / M\rfloor} \Omega \gamma^{j+n M}$ are the postcursor and precursor ISI with respect to the current transmit symbol $b(i)$, respectively.

We use variable $\beta_{j}$ to measure the ratio between the average power of the $j$ th path and $I^{(j)}$, which is defined as

$$
\begin{aligned}
\beta_{j} & \equiv \frac{E\left\{\alpha_{j}^{2}\right\}}{I^{(j)}}=\frac{\Omega \gamma^{j}}{I^{(j)}} \\
& =\frac{\Omega \gamma^{j}}{\sum_{m=1}^{\lfloor j / M\rfloor} \Omega \gamma^{j-m M}+\sum_{n=1}^{\lfloor(L-j) / M\rfloor} \Omega \gamma^{j+n M}} \\
& =\frac{1}{\sum_{m=1}^{\lfloor j / M\rfloor} \gamma^{-m M}+\sum_{n=1}^{\lfloor(L-j) / M\rfloor} \gamma^{n M}} .
\end{aligned}
$$

We get from (39) that

$$
\begin{aligned}
\beta_{0} & =\cdots=\beta_{M-1}>\beta_{M}=\cdots \\
& =\beta_{2 M-1}>\cdots>\beta_{(K-1) M}=\cdots=\beta_{K M-1}
\end{aligned}
$$

which suggests a way to divide all $L$ path components into $K$ disjoint groups so that elements with the same value of $\beta$ belong to the same group. For example, group 1 has the 0 th to the $(M-$ 1 )th elements, group 2 has the $M$ th to the $(2 M-1)$ th elements, and so on.

The following lemma will be needed in deriving the fast search algorithm.

Lemma 1: If $S_{1}, S_{2}, I_{1}$, and $I_{2}$ are all positive numbers, then

$$
\frac{S_{2}}{I_{2}}<\frac{S_{1}}{I_{1}} \Longleftrightarrow \frac{S_{2}}{I_{2}}<\frac{S_{1}+S_{2}}{I_{1}+I_{2}}<\frac{S_{1}}{I_{1}} .
$$

It is straightforward to verify this lemma. The following proposition determines the optimal codeword length when the codeword length does not excess $M$.

Proposition 2: When $0<l \leq M, M=\arg \max _{0<l \leq M} \bar{\nu}^{(l)}$. Proof: Let $l=k \leq M$. We can simplify $\bar{\nu}^{(k)}$ as

$$
\begin{aligned}
\bar{\nu}^{(k)} & =\frac{E\left\{\left(\sum_{j=0}^{k-1} \alpha_{j}\right)^{2}\right\}}{\sum_{j=0}^{k-1} I^{(j)}} \\
& =\frac{E\left\{\sum_{j=0}^{k-1} \alpha_{j}^{2}+\sum_{i, j=0 ; i \neq j}^{k-1} \alpha_{i} \alpha_{j}\right\}}{I^{(0)} \sum_{j=0}^{k-1} d^{2 j}} \\
& =\frac{\Omega\left(\sum_{j=0}^{k-1} d^{2 j}+\frac{\pi}{2} \sum_{i=1}^{k-1} \sum_{j=1}^{i} d^{i+j-1}\right)}{I^{(0)} \sum_{j=0}^{k-1} d^{2 j}} \\
& =\beta_{0}+\beta_{0} \frac{\pi}{2} \frac{\sum_{i=1}^{k-1} \sum_{j=1}^{i} d^{i+j-1}}{\sum_{j=0}^{k-1} d^{2 j}} \\
& =\beta_{0}+\beta_{0} \frac{\pi}{2} g(k)
\end{aligned}
$$

where $d=\gamma^{1 / 2}<1$ and $g(k)=$ $\left(\sum_{i=1}^{k-1} \sum_{j=1}^{i} d^{i+j-1}\right) /\left(\sum_{j=0}^{k-1} d^{2 j}\right) \quad$ is a positive function of code length $k$. In Section X-A, we will show that $g(k)$ is a monotonically increasing function of $k$ for $1 \leq k \leq M$. Thus, the maximum SIR $\bar{\nu}^{(k)}$ must occur at $k=M$.

Next, we consider the case with code length $l>M$. We can represent $l$ as

$$
l=k M+\bar{l}
$$

where $k$ and $1 \leq \bar{l} \leq M$ are positive integers defined as $k \equiv$ $\lceil(l-M) / M\rceil$ and $\bar{l} \equiv l-k M$, respectively. Then, the output SIR can be rewritten as

$$
\bar{\nu}^{(l)}=\frac{S(l)}{I(l)}=\frac{S(k M)+\Delta S(\bar{l})}{I(k M)+\Delta I(\bar{l})}
$$

where $S(k M) \equiv E\left\{\left(\sum_{j=0}^{k M-1} \alpha_{j}\right)^{2}\right\}$ and $I(k M) \equiv \sum_{j=0}^{k M-1} I^{(j)}$ are the signal power and interference power obtained by combining the first $k M$ channel taps, $\Delta S(\bar{l}) \equiv S(k M+\bar{l})-$ $S(k M)=E\left\{\sum_{j=k M}^{l-1} \alpha_{j}^{2}+2 \sum_{i=0}^{k M-1} \sum_{j=k M}^{l-1} \alpha_{i} \alpha_{j}\right\}$ and $\Delta I(\bar{l}) \equiv \sum_{j=k M}^{l-1} I^{(j)}$ are the amounts of increased signal and noise power due to the extension of code length from $k M$ to $k M+\bar{l}$, respectively. Please notice that both $\Delta S$ and $\Delta I$ are functions of $k$ and $\bar{l}$, i.e., $\Delta S=\Delta S(k, \bar{l})$ and $\Delta I=\Delta I(k, \bar{l})$. Here, we ignore the variable $k$ for notation simplicity. 
Lemma 2: For fixed $k$, we have

$$
\frac{\Delta S(\bar{l})}{\Delta I(\bar{l})} \equiv \frac{E\left\{\sum_{j=k M}^{l-1} \alpha_{j}^{2}+2 \sum_{i=0}^{k M-1} \sum_{j=k M}^{l-1} \alpha_{i} \alpha_{j}\right\}}{\sum_{j=k M}^{l-1} I^{(j)}}
$$

is a monotonic increasing function of $\bar{l}$ when $1 \leq \bar{l} \leq M$.

Proof: See Section X-C.

The upper bound on $\bar{\nu}^{(l)}$ is given in the following proposition.

Proposition 3: If $k M \leq l \leq(k+1) M$, the output SIR, $\bar{\nu}^{(l)}$, is upper bounded by either $\bar{\nu}^{(k M)}$ or $\bar{\nu}^{((k+1) M)}$.

Proof: See Section X-D.

The upper bound on $\bar{\nu}^{(l)}$ in Proposition 3 indicates that the maximum output SIR must be bounded by $\bar{\nu}^{(k \cdot M)}$, $k=1, \ldots, K$, i.e.,

$$
\max _{0<l \leq L} \bar{\nu}^{(l)} \leq \max _{0<k \leq K} \bar{\nu}^{(k \cdot M)} .
$$

Thus, we are led to the following fast search algorithm.

Proposition 4: A fast search algorithm to determine an optimal codeword length $L_{\mathrm{opt}}$ can be written as

$$
L_{\mathrm{opt}}=\arg \max _{0<l \leq L} \bar{\nu}^{(l)}=M \cdot \arg \max _{0<k \leq K} \bar{\nu}^{(k M)}
$$

and the corresponding maximum output SIR is

$$
\bar{\nu}^{\left(L_{\mathrm{opt}}\right)}=\frac{E\left\{\left(\sum_{j=0}^{L_{\mathrm{opt}}-1} \alpha_{j}\right)^{2}\right\}}{\sum_{j=0}^{L_{\mathrm{opt}}-1} I^{(j)}} .
$$

As compared with the exhaustive search algorithm in (38), the fast algorithm reduces the search number by a factor of $M$.

Another possible criterion for CLO is to consider both AWGN and ISI jointly. That is, we can maximize the output SINR, i.e.,

$$
\hat{L}_{\mathrm{opt}}=\arg \max _{0<l \leq L}\left(\frac{E\left\{\left(\sum_{j=0}^{l-1} \alpha_{j}\right)^{2}\right\}}{l \cdot\left(N_{0} / 2\right)+\sum_{j=0}^{l-1} I^{(j)}}\right) .
$$

The fast search algorithm in Proposition 4 may not give the maximum output SINR, especially in the low SNR environment. We will show in Section VIII that the performance gap between the two criteria as specified in (45) and (47) is small, even in the presence of high noise power. In addition, the difference approaches zero asymptotically as SNR increases.

\section{DESIGN OF MMSE RECEIVER}

As the data rate increases, the performance degrades since the peak received signal of a target symbol suffers more ISI from its preceding and succeeding symbols. Although CLO can mitigate ISI by adjusting the code length, it may not suppress ISI sufficiently in a high data rate case. In this section, we examine the use of an MMSE receiver to suppress ISI furthermore.

When we encode symbol $b(i)$ with an $l$-chip codeword $\mathbf{c}^{(l)}$, the signal of $b(i)$ will spread over $L+l-1$ received chips. The received signal to decode $b(i)$ contains contributions from its $L_{0}$ preceding and $L_{1}(\equiv\lfloor(l-1) / M\rfloor)$ succeeding symbols. Mathematically, input symbol $b(i)$ and output peak signal $r_{l-1}^{(l)}(i)$ are related via

$$
r_{l-1}^{(l)}(i)=\sum_{j=-L_{1}}^{L_{0}} \bar{h}_{j} b(i-j)+n_{l-1}^{(l)}(i)
$$

where $\bar{h}_{j} \equiv h_{l+j M-1}^{(l)}$ and $r_{l-1}^{(l)}(i)$ and $n_{l-1}^{(l)}(i)$ represent the $l$ th components of $\mathbf{r}_{l}(i)$ and $\mathbf{n}_{l}(i)$, respectively. Equation (48) can be represented in matrix form as $\tilde{\mathbf{r}}(i)=\tilde{\mathbf{H}} \tilde{\mathbf{b}}(i)+\tilde{\mathbf{n}}(i)$, where

$$
\tilde{\mathbf{H}} \equiv\left[\begin{array}{ccccc}
\bar{h}_{L_{0}} & \cdots & \bar{h}_{-L_{1}} & & \mathbf{0} \\
& \ddots & \ddots & \ddots & \\
\mathbf{0} & & \bar{h}_{L_{0}} & \cdots & \bar{h}_{-L_{1}}
\end{array}\right]
$$

$\tilde{\mathbf{b}}(i)=\left[b\left(i-L_{0}-L_{1}\right), \ldots, b(i), \ldots, b\left(i+L_{0}+L_{1}\right)\right]^{T}$, $\tilde{\mathbf{r}}(i)=\left[r_{l-1}^{(l)}\left(i-L_{1}\right), \ldots, r_{l-1}^{(l)}\left(i+L_{0}\right)\right]^{T}$ and $\tilde{\mathbf{n}}(i)=$ $\left[n_{l-1}^{(l)}\left(i-L_{1}\right), \ldots, n_{l-1}^{(l)}\left(i+L_{0}\right)\right]^{T}$. Note that $\tilde{\mathbf{r}}(i)$ and $\tilde{\mathbf{n}}(i)$ are the received signal and noise vectors, respectively.

Then, the MMSE receiver $\mathbf{w}$ for data symbol $b(i)$ can be written as $\mathbf{w}=\mathbf{R}_{\tilde{\mathbf{r}}}^{-1} \mathbf{p}$, where $\mathbf{R}_{\tilde{\mathbf{r}}} \equiv E\left\{\tilde{\mathbf{r}}(i) \tilde{\mathbf{r}}(i)^{T}\right\}=\tilde{\mathbf{H}} \tilde{\mathbf{H}}^{T}+$ $\left(N_{0} / 2\right) \mathbf{I}_{L_{0}+L_{1}+1}$ is the autocorrelation matrix of $\tilde{\mathbf{r}}(i)$ and $\mathbf{p} \equiv$ $E\{\tilde{\mathbf{r}}(i) b(i)\}=\left[\bar{h}_{-L_{1}}, \ldots, \bar{h}_{L_{0}}\right]^{T}$ is the cross correlation between $\tilde{\mathbf{r}}(i)$ and $b(i)$. Please note that we treat $\bar{h}_{i},-L_{1} \leq i \leq L_{0}$, as fixed variables by assuming that channel coherent time is long enough so that the channel is invariant for a block of data symbols. Thus, $b(i)$ can be estimated as $\hat{b}(i)=\operatorname{sign}\left\{\mathbf{w}^{T} \tilde{\mathbf{r}}(i)\right\}$. As compared with the CPP receiver without MMSE, which is a single tap filter, the MMSE receiver is a finite impulse response (FIR) filter of length $L_{0}+L_{1}+1$. Thus, it has higher decoding complexity. Furthermore, the MMSE receiver demands training symbols to estimate $\bar{h}_{-L_{1}}, \ldots, \bar{h}_{L_{0}}$ at the cost of a decreased data rate.

\section{CONSIDERation of FCC POWER SPeCtral Mask}

The U.S. FCC allows UWB signals to occupy a huge range of frequency spectrum that overlaps with those used in existing narrow band radio services today [8]. To coexist with other radio signals with little interference, a power spectrum mask is enforced to regulate the output power spectral density (PSD) of the UWB transmitter [8]. Since the proposed precoding scheme alters the output signal shape, the PSD at the transmit antenna may exceed the FCC mask. This concern is addressed in this section. In the following, we derive the output PSD of the CPP-UWB system and then explain how to maintain the FCC regulation on the output PSD when the transmit symbol is encoded by the channel phase.

We consider the case where the symbol interval is shorter than the channel response and the channel length is an integer multiple of the symbol interval, i.e., $L=K M$ with $K$ being a positive integer. When the energy per pulse is equal to $E_{s}$, a general transmitted signal can be written as

$$
\begin{aligned}
& x_{s}(t)=\sqrt{\frac{E_{s}}{L}} \sum_{l=-\infty}^{\infty} \sum_{k=0}^{K-1} \sum_{j=0}^{M-1} b(l-k) \\
& \quad \times p_{L-1-k M-j} w_{s}(t-l M \Delta-j \Delta)=w_{s}(t) \otimes x_{1}(t)
\end{aligned}
$$


where " $\otimes$ " is the convolution operator and

$$
\begin{aligned}
x_{1}(t)=\sqrt{\frac{E_{s}}{L}} \sum_{l=-\infty}^{\infty} \sum_{\substack{k=0\\
}}^{K-1} \sum_{j=0}^{M-1} b(l-k) \\
\quad \times p_{L-1-k M-j} \delta(t-l M \Delta-j \Delta) .
\end{aligned}
$$

Since $x_{1}(t)$ is an infinite random sequence, we truncate it by

$$
x_{1}^{(T)}(t)= \begin{cases}x_{1}(t), & -T-\epsilon \leq t \leq T-\epsilon \\ 0, & \text { elsewhere }\end{cases}
$$

where $0<\epsilon \ll 1$ and the value of $T$ is selected to be an integer multiple of the symbol interval, i.e., $T=T_{s} \alpha$, where $\alpha$ is a positive number. Then, $x_{1}^{(T)}(t)$ can be explicitly expressed as

$$
\begin{aligned}
x_{1}^{(T)}(t)=\sqrt{\frac{E_{s}}{L}} \sum_{l=-\alpha}^{\alpha-1} \sum_{\substack{k=0 \\
\times}}^{K-1} \sum_{j=0}^{M-1} b(l-k) \\
\quad \times p_{L-1-k M-j} \delta(t-l M \Delta-j \Delta) .
\end{aligned}
$$

The time-averaged autocorrelation function of $x_{1}^{(T)}(t)$ can be found as

$$
\begin{aligned}
\frac{1}{2 T} E\left\{x_{1}^{(T)}\left(t_{1}\right) x_{1}^{(T)}\left(t_{2}\right)\right\} & =\frac{E_{s}}{2 T_{s} L \alpha} 2 \alpha M K \delta\left(t_{1}-t_{2}\right) \\
& =\frac{E_{s}}{T_{s}} \delta(\tau)
\end{aligned}
$$

where $\tau=t_{1}-t_{2}$. Consequently, the PSD of $x_{1}(t), S_{x_{1}}(f)$, is equal to

$$
\begin{aligned}
S_{x_{1}}(f) & =\lim _{T \rightarrow \infty} \mathbb{F}\left\{\frac{1}{2 T} E\left\{x_{1}^{(T)}\left(t_{1}\right) x_{1}^{(T)}\left(t_{2}\right)\right\}\right\} \\
& =\lim _{\alpha \rightarrow \infty} \mathbb{F}\left\{\frac{E_{s}}{T_{s}} \delta(\tau)\right\}=\frac{E_{s}}{T_{s}}
\end{aligned}
$$

where $\mathbb{F}\{\cdot\}$ is the Fourier transform operator. Note that the PSD of $x_{s}(t)$ is computed as

$$
S_{x_{s}}(f)=S_{x_{1}}(f)\left|W_{s}(f)\right|^{2}=\frac{E_{s}}{T_{s}}\left|W_{s}(f)\right|^{2}
$$

where $W_{s}(f)$ is the Fourier transform of $w_{s}(t)$. We see from (56) that the output PSD is proportional to $\left|W_{s}(f)\right|^{2}$. In addition, it can be easily shown that the output PSD for the CPP-UWB system with the optimized codeword length introduced in Section V is the same as (56).

Given a fixed data rate, the admissible energy per pulse $E_{s}$ is limited by enforcing the FCC power mask. To pump as much power as possible to improve the system performance while maintaining the FCC constraint on the output PSD, it is important to design a proper pulse waveform. The pulse waveform design problem for the CPP-UWB system is an interesting problem for future extension. We refer interested readers to [16] and references therein for detailed discussion on the UWB pulse waveform design. It is worthwhile to point out that, to maintain the power spectrum mask, the transmit power should be reduced for a fixed pulse waveform while the symbol interval is shortened. As a result, the received signal power decreases as well. If we want to maintain the same SNR level as that of the low data rate system, the noise figure at the high data rate receiver

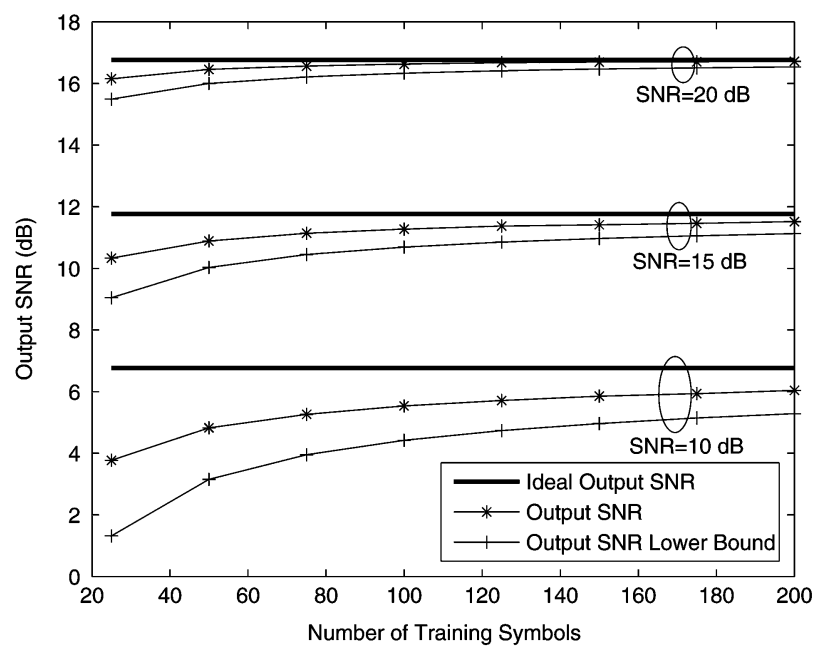

Fig. 3. Output SNR versus the number of training symbols, where $\Delta=0.5$ ns, $\Gamma=20.5 \mathrm{~ns}(\mathrm{CM} 3)$, and $L=240$.

should be further reduced, i.e., more attention is needed for its hardware implementation.

\section{Simulation Results}

Simulations are conducted in this section to test the performance of the proposed CPP scheme in an UWB system. We demonstrate the phase estimation scheme and the corresponding lower bound on SNR as provided in Section IV in the first example. Then, we compare the bit error rate (BER) performance for the CPP-UWB system at different temporal resolutions, i.e., using UWB pulses with different pulse width in the second example. The BER performance can be further improved by applying CLO and/or the MMSE receiver, which is shown in the third example. Next, we verify the argument in Section V that the channel length is an integer multiple of the symbol interval. Finally, we compare the feedback overhead and the BER performance between CPP and TRP, and show that the SINR degradation due to the fast search algorithm diminishes monotonically as the input SNR increases in the last example. In order to stay within the FCC power spectrum mask, the transmit power of the CPP-UWB system is adjusted according to different data rates. It is assumed that the noise figure reduction at the receiver utilized for the high data rate reception is possible so that we can compare the system performance for different data rates at the same SNR level.

Example 1: Effect of Proposed Phase Estimation Algorithm: We study the impact of training overhead on the performance of the phase estimation algorithm proposed in Section IV. The system parameters are $\Delta=0.5 \mathrm{~ns}, \Gamma=20.5 \mathrm{~ns}$ (CM3), $L=$ 240 , and three input SNR values $(10,15$, and $20 \mathrm{~dB})$ are considered. Thus, the value of $\eta$ is computed as

$$
\eta=(240 * 0.5) / 20.5 \approx 5.85 .
$$

The results are obtained by averaging over 1000 channel realizations. Fig. 3 shows the output SNR at the peak received signal and its corresponding lower bound as a function of the number $N$ of training symbols. The ideal output SNR without any phase 


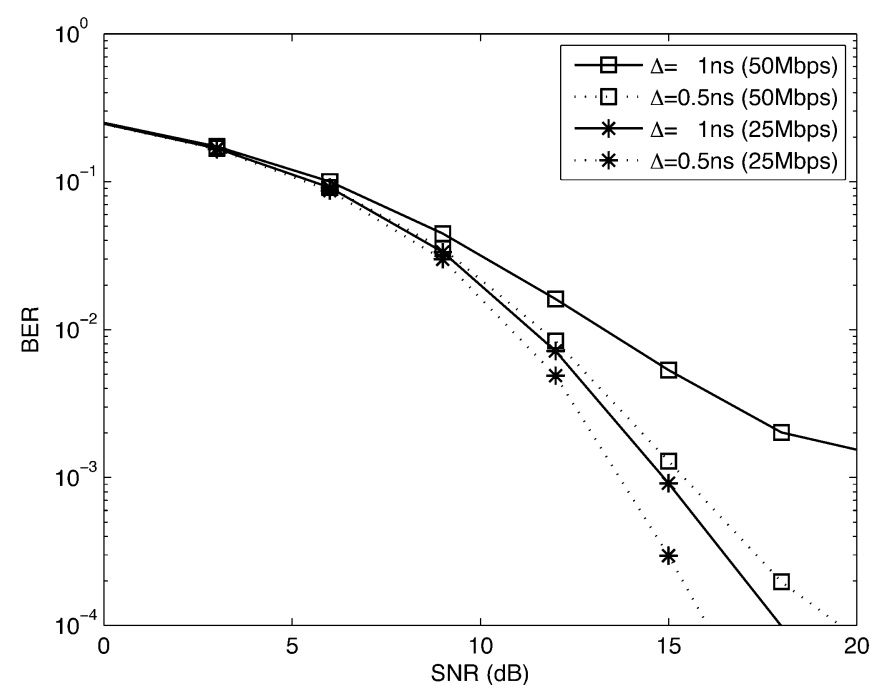

Fig. 4. BER performance at different temporal resolutions for various data rates, where $L=120,240$ for $\Delta=1,0.5 \mathrm{~ns}$, respectively, and $\Gamma=20.5 \mathrm{~ns}$ (CM3).

mismatch is also shown as a performance benchmark. We see from Fig. 3 that, for a given input SNR, the phase estimation can be improved using more training symbols. However, a larger number of training symbols leads to higher overhead and, thus, degrades the data rate. For a fixed number of training symbols, better system performance is expected when the noise power is smaller. The lower bound predicts the worst system performance for a given number of training symbols. We may adjust the number of training symbols to meet some performance requirement while keeping the data rate as high as possible. It is observed that the gap between the true output SNR and the corresponding lower bound decreases as $N$ or the input SNR increases. This is because the upper bound for $Q(x)$ gets tight as $x$ increases.

In addition, there is a $3.1 \mathrm{~dB}$ gap between the input SNR (or the ideal output SNR of TRP) and the ideal output SNR of CPP-UWB in Fig. 3 since not all the channel information is utilized at the CPP transmitter. This degradation can be verified by computing the degradation factor defined in (16), i.e.,

$$
\left.\eta \approx \frac{\pi\left(1-e^{-\eta / 2}\right)}{\eta\left(1+e^{-\eta / 2}\right)}\right|_{\eta=5.85}=0.4821=-3.17 \mathrm{~dB}
$$

which corroborates the approximations given in (9) and (12).

Example 2: BER Performance at Different Multipath Resolutions: The BER performance of the CPP-UWB system with different multipath resolutions due to different pulse rates is studied in this example. The UWB pulse width is selected to be 1 and $0.5 \mathrm{~ns}$, and the effective numbers of channel taps are assumed to be 120 and 240, accordingly, for CM3. Two data rates are considered: 50 and $25 \mathrm{Mbps}$. The corresponding BER curves at different SNR values shown in Fig. 4 are averaged over 1000 channel realizations. We see that a higher data rate can be achieved by shortening the symbol interval. However, more serious ISI occurs and the system performance degrades in this case. Although better performance can be achieved using a narrower pulse (i.e., higher multipath resolution), this also increases the feedback overhead and the hardware implementation

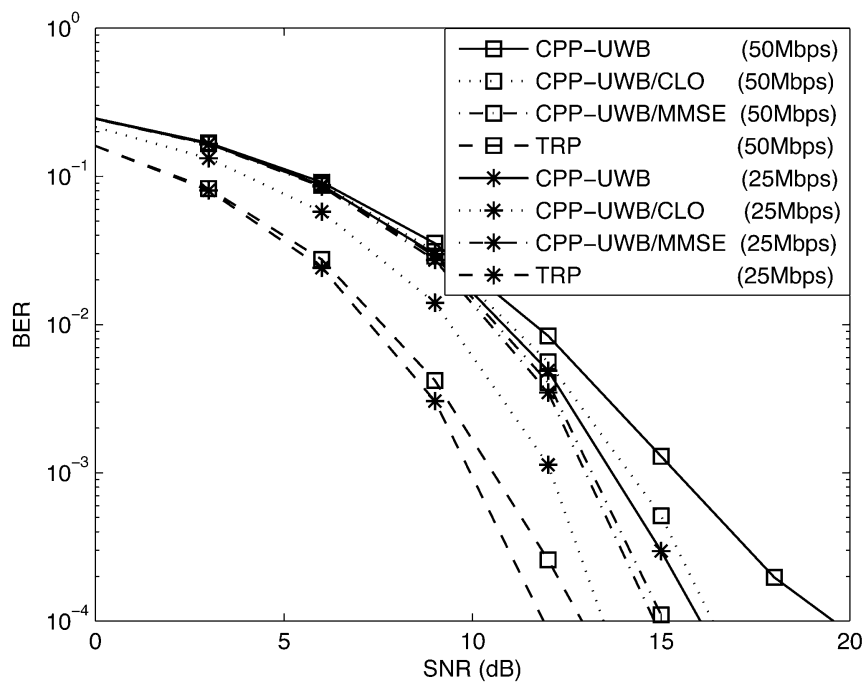

Fig. 5. BER performance improvement with different ISI suppression schemes at different data rates, where $\Delta=0.5 \mathrm{~ns}, \Gamma=20.5 \mathrm{~ns}(\mathrm{CM} 3)$, and $L=240$.

cost. Thus, it is important to choose a suitable pulse rate that can achieve the desired system performance while keeping reasonable feedback overhead.

Example 3: Residual ISI Suppression: We show that the system performance can be further improved using the CLO technique and the MMSE receiver as discussed in Sections V and VI, respectively, in this example. The system parameters are the same as those in Example 2 except that the UWB pulse width is set to $0.5 \mathrm{~ns}$ only. To simplify the discussion, estimates for $\bar{h}_{-L_{1}}, \ldots, \bar{h}_{L_{1}}$ in the MMSE receiver are assumed to be perfect and the code length for CPP-UWB with the MMSE receiver is $L$. We plot the BER curve as a function of SNR for the CPP-UWB system with CLO or the MMSE receiver at two data rates (i.e., 50 and $25 \mathrm{Mbps}$ ) in Fig. 5. In addition, the corresponding BER curves for TRP and the conventional CPP without CLO are plotted for the purpose of benchmarking. The MMSE receiver gives similar performance at these two different data rates. However, when the codeword length is fixed, the increased data rate will have more overlapped received signals so that the complexity of the MMSE receiver increases as well. On the other hand, the CLO technique enhances the performance and maintains a simple receiver structure. The performance of the CLO method using the proposed fast search algorithm is worse than the MMSE receiver at the rate of $50 \mathrm{Mbps}$, but better than the MMSE receiver at the rate of $25 \mathrm{Mbps}$ due to less overlapped symbols by adjusting the codeword length. When the noise power is stronger than ISI (e.g., the case with input SNR less than $5 \mathrm{~dB}$ ), the performance gap between CPP-UWB and TRP is about $3 \mathrm{~dB}$, which can be inferred from (58). When ISI grows (say, due to the increase of the data rate or the signal power), the gap becomes larger since TRP suppresses ISI more efficiently than CPP. Although TRP minimizes the symbol detection error probability for a given SNR level at the cost of higher feedback overhead and a higher resolution ADC, the performance gap between CPP-UWB/CLO and TRP at the data rate of $25 \mathrm{Mbps}$ decreases asymptotically as the noise power decreases since CLO reduces the ISI at the receiver output. 


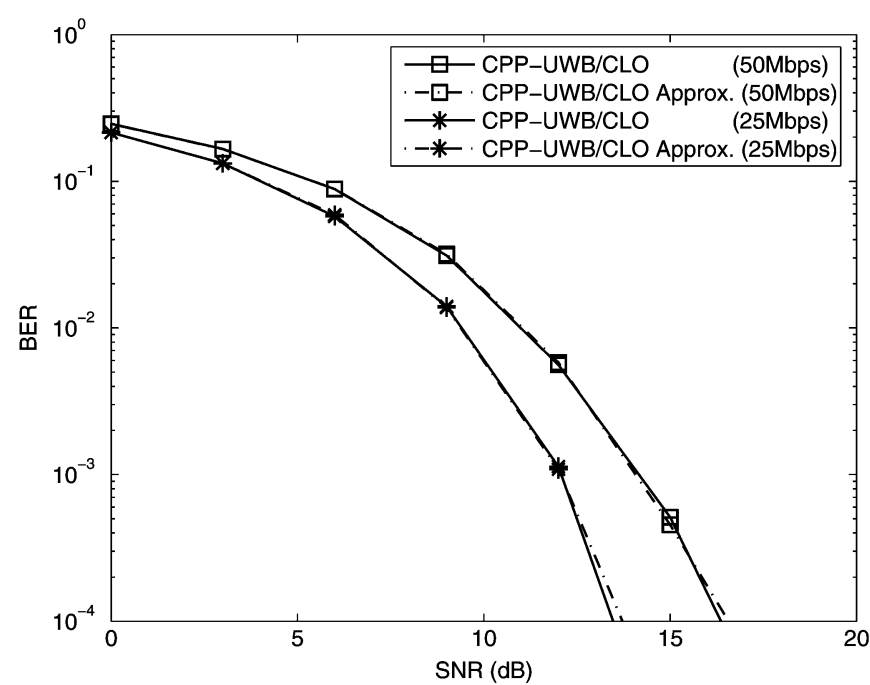

Fig. 6. BER performance comparison between two systems using the approximated and the real channels, where $\Delta=0.5 \mathrm{~ns}, \Gamma=20.5 \mathrm{~ns}$ (CM3).

Example 4: Effect of Channel Length Approximation and Comparison of Fast and Exhaustive Search for CLO: The length of channel is assumed to be 240 chips long in Example 3, where taps whose power ratio to the first tap greater or equal to $e^{-\eta}=e^{-5.85} \approx 2.88 * 10^{-3}$ are included. However, the real channel length may be longer or shorter than 240. It was claimed that we can pad zeros or truncate the channel tail to satisfy the channel length requirement in Section V. We would like to verify this claim here. Suppose that the true channel length can be modeled as a random integer uniformly distributed between 205 and 287 . These numbers correspond to the power ratio of the last tap and the first tap equal to $e^{-205 /(20.5 * 0.5)} \approx 6.74 * 10^{-3}$ and $e^{-287 /(20.5 * 0.5)} \approx 9.1 * 10^{-4}$, respectively. As suggested in Section V, the channel can be truncated or zero-padded so that the modified channel length is equal to 240 chips. The optimal codeword length that maximizes the output SIR can be found by exhaustive search algorithm. The other system parameters are kept the same as those in the Example 3. Fig. 6 shows BER curves for the CPP-UWB system with a fixed channel length $L=240$ and a variable channel length under various data rates. As shown in the figure, the truncation or zero-padding of the original channel does not change the performance of the system much. Besides, the use of the exhaustive search algorithm and the fast search algorithm for CLO does not make any visible difference. Consequently, we can adjust the length of the UWB channel when the power of taps at the channel tail is small enough. Furthermore, the computational power for the optimal code length can be saved by applying the fast search algorithm.

Example 5: Performance Comparison Between CPP and $P P R$ : In this example, we compare the feedback overhead of CPP-UWB with CLO and conventional PPR with a single-finger RAKE receiver. In order to perform fair comparison between these two different systems, we focus on the case where the two systems have about the same peak power. Once the optimal code length for CPP-UWB is determined using the fast algorithm, we can use (22) to compute the number of channel taps
TABLE I

FEEDBACK OVERHEAD REQUIRED BY CPP AND PPR TO ACHIEVE THE SAME PEAK POWER

\begin{tabular}{|c|c|c|}
\hline & 50Mbps & 25Mbps \\
\hline CPP Code Length & 40 & 80 \\
\hline PPR Feedback Tap Number & 28 & 41 \\
\hline
\end{tabular}

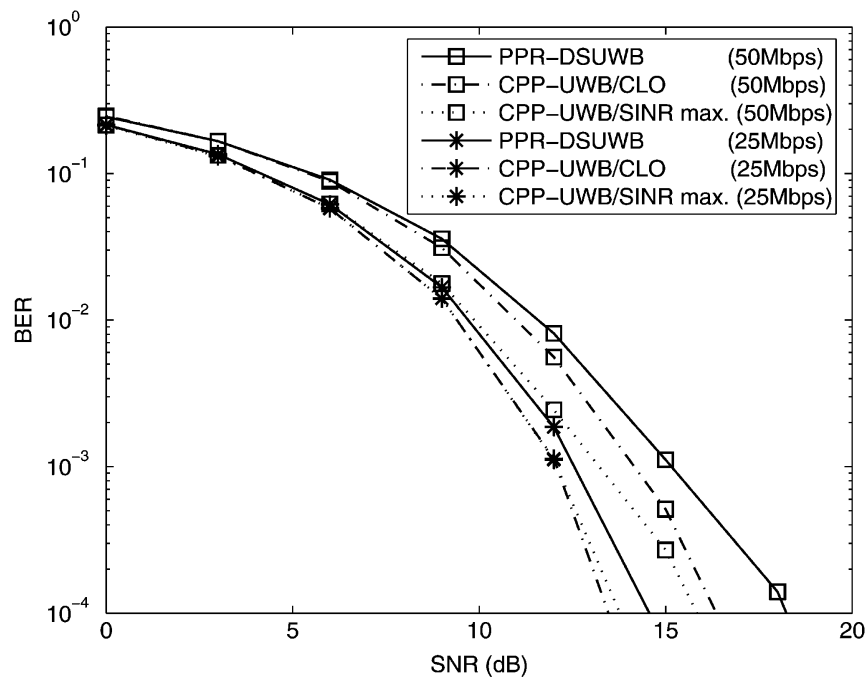

Fig. 7. Performance comparison between PPR-UWB and CPP-UWB with different code lengths, where $\Delta=0.5 \mathrm{~ns}, L=240, \Gamma=20.5 \mathrm{~ns}(\mathrm{CM} 3)$.

required by the PPR transmitter to generate roughly the same peak power. The system parameters are the same as those in Example 4. Table I shows the numbers of the fed back phases and taps required for CPP and PPR, respectively. If an 10-bit analog-to-digital converter (ADC) is used to digitize each tap, the receiver of PPR will send a total number of $280(=28 * 10)$ and $410(=41 * 10)$ bits back to the transmitter for data rates of 50 and $25 \mathrm{Mbps}$, respectively. It is clear that PPR requires more feedback overhead than CPP.

Next, we test the BER performance of these two systems with the feedback overhead specified in Table I. To demonstrate the SINR degradation using the proposed fast algorithm, we draw the performance curves for the CPP-UWB system whose codeword length gives the maximum output SINR. The results are shown in Fig. 7. Even though the output SINR of these two systems may not be the same with the amount of feedback given in (22), the discrepency is small. Thus, the feedback overhead comparison in Section III still holds. It is also observed in Fig. 7 that the performance gap between systems using different code length optimization criteria is small, and it decreases as SNR increases.

This can be intuitively explained as follows. When the system suffers from strong noise power, the performance is less sensitive to the interference power due to different code lengths. In addition, the code length obtained by fast search yields the maximum output SINR asymptotically as input SNR increases, which corroborates our derivation and argument as in Section V. To illustrate this point better, we plot the output SINR as a function of the code length and the input SNR for a fixed data rate 


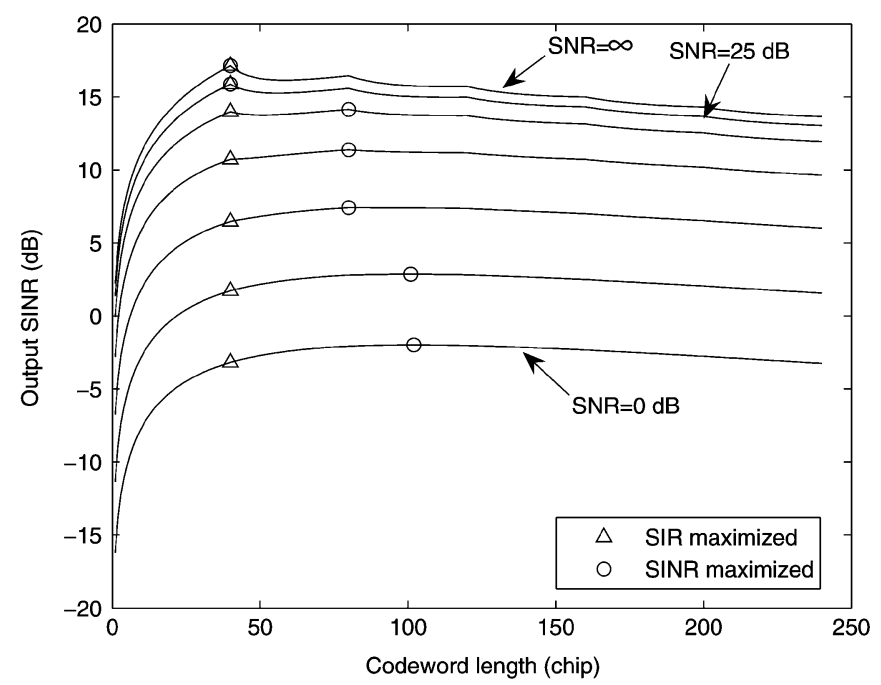

Fig. 8. Output SINR with different codeword lengths at different input SNR values, where $\Delta=0.5 \mathrm{~ns}, L=240, \Gamma=20.5 \mathrm{~ns}$ (CM3).

at $50 \mathrm{Mbps}$ and using the same parameters as previous examples in Fig. 8. The triangular and circle marks denote the output SINR for different code length selection schemes. The lower six curves represent cases that input SNR is equal to 0 to $25 \mathrm{~dB}$ with a step size of $5 \mathrm{~dB}$. The top curve is the case where the noise power is zero, i.e., the output SIR. We see that the SINR gap between these two code length selection schemes is small even in the low SNR region. However, the computational complexity of finding the optimal code length by the full search algorithm is reduced by a factor of 40 times when the fast search algorithm is used instead.

\section{CONCLUSION}

A new UWB transceiver that encodes each transmit data symbol with the channel phase information, called the CPP-UWB system, was proposed in this work. It was shown that CPP-UWB is computationally more efficient than the conventional TRP-based system. It demands lower hardware complexity which is critical in the UWB system design. The channel phase can be estimated using training symbols, and the performance of the estimated phase can be evaluated using the derived lower bound on the output SNR. By exploiting the power concentration of the received signal, we can shorten the symbol interval for high data rate transmission with little performance degradation. The performance can be further improved for the high rate communication by optimizing the codeword length to maximize the output SIR. Even though a closed form solution may not be obtainable due to the nonlinear nature of the optimization problem, we develop a fast search scheme to reduce the computational load of the exhaustive search algorithm. The proposed algorithm degrades a little from the maximum output SINR in the low SNR case, and it converges to the true maximum output SINR as the noise power decreases. As compared to the PPR system, the CPP-UWB system demands less feedback overhead and achieves better performance when the two systems have about the same peak power level.

\section{APPENDIX}

\section{A. Proof of the Monotonic Increasing Property of $g(k)$}

In this section, we show that $g(k)$ is a monotonic increasing function for $1 \leq k \leq M$. By using

$$
\begin{aligned}
g(k+1) & =\frac{\sum_{i=1}^{k} \sum_{j=1}^{i} d^{i+j-1}}{\sum_{j=0}^{k} d^{2 j}} \\
& =\frac{\sum_{i=1}^{k-1} \sum_{j=1}^{i} d^{i+j-1}+\sum_{j=1}^{k} d^{k+j-1}}{\sum_{j=0}^{k-1} d^{2 j}+d^{2 k}}
\end{aligned}
$$

and the following inequality (see Section X-B for proof)

$$
\frac{\sum_{j=1}^{i} d^{i+j-1}}{\sum_{j=0}^{k-1} d^{2 j}}<1, \quad \forall 1 \leq i \leq(k-1)
$$

$g(k)$ can be upper bounded by

$$
g(k)=\frac{\sum_{i=1}^{k-1} \sum_{j=1}^{i} d^{i+j-1}}{\sum_{j=0}^{k-1} d^{2 j}}<\sum_{i=1}^{k-1} 1=k-1 .
$$

Next, consider another lower bound for the following term:

$$
\frac{\sum_{j=1}^{k} d^{k+j-1}}{d^{2 k}}=\sum_{j=1}^{k} d^{j-k-1}>\sum_{j=1}^{k} 1=k .
$$

Thus, by applying Lemma 1 to (60) and (61), we conclude that

$$
\begin{aligned}
g(k+1) & =\frac{\sum_{i=1}^{k-1} \sum_{j=1}^{i} d^{i+j-1}+\sum_{j=1}^{k} d^{k+j-1}}{\sum_{j=0}^{k-1} d^{2 j}+d^{2 k}} \\
& >g(k)
\end{aligned}
$$

which suggests that $g(k)$ is a monotonic increasing function of $k$ when $1 \leq k \leq M$.

\section{B. Proof of (59)}

Consider the following equation:

$$
\begin{aligned}
\sum_{j=0}^{k-1} d^{2 j} & -\sum_{j=1}^{i} d^{i+j-1} \\
= & (\underbrace{1+d^{2}+\cdots+d^{2 k-2}}_{k-\text { element }}) \\
& -(\underbrace{d^{i}+d^{i+1}+\cdots+d^{2 i-1}}_{i-\text { elemnet }}) .
\end{aligned}
$$

When $i(<k-1)$ is an even number, which can be represented as $i=2 m$ where $m$ is a positive integer, (63) can be simplified 
as

$$
\begin{aligned}
\sum_{j=0}^{k-1} & d^{2 j}-\sum_{j=1}^{i} d^{i+j-1} \\
= & \left(1+d^{2}+\cdots+d^{2 k-2}\right) \\
& -\left(d^{2 m}+d^{2 m+1} \cdots+d^{4 m-1}\right) \\
= & \underbrace{1+d^{2}+\cdots+d^{2 m-2}}_{m-\text { element }} \\
& +d^{4 m+2}+\cdots+d^{2 k-2}-(\underbrace{d^{2 m+1}+\cdots+d^{4 m-1}}_{m-\text { element }}) \\
= & \left(1-d^{2 m+1}\right)\left(1+d^{2}+\cdots+d^{2 m-2}\right) \\
& +d^{4 m+2}+\cdots+d^{2 k-2}>0
\end{aligned}
$$

since $0<d<1$. In addition, by a method similar to the above, we can prove that the inequality still holds even for an odd number of $l$. Since both $\sum_{j=0}^{k-1} d^{2 j}$ and $\sum_{j=1}^{i} d^{i+j-1}$ are positive and $\sum_{j=0}^{k-1} d^{2 j}-\sum_{j=1}^{i} d^{i+j-1}>0$, we conclude that (59) is true.

\section{Proof of Lemma 2}

Here, we decompose $\Delta S(\bar{l})$ into two parts as

$$
\begin{aligned}
\Delta S(\bar{l}) & =E\left\{\sum_{j=k M}^{k M+\bar{l}-1} \alpha_{j}^{2}+2 \sum_{i=0}^{k M-1} \sum_{j=k M}^{k M+\bar{l}-1} \alpha_{i} \alpha_{j}\right\} \\
& =\Delta S_{1}(\bar{l})+\Delta S_{2}(\bar{l})
\end{aligned}
$$

where $\Delta S_{1}(\bar{l})=E\left\{\sum_{j=k M}^{k M+\bar{l}-1} \alpha_{j}^{2}\right\}$ and $\Delta S_{2}(\bar{l})=E\left\{2 \sum_{i=0}^{k M-1}\right.$ $\left.\sum_{j=k M}^{k M+\bar{l}-1} \alpha_{i} \alpha_{j}\right\}=(\pi \Omega / 2) \sum_{i=0}^{k M-1} \cdot \sum_{j=k M}^{k M+\bar{l}-1} d^{i+j}$. Thus, we have

$$
\begin{aligned}
& \frac{\Delta S(\bar{l})}{\Delta I(\bar{l})} \\
& \quad=\frac{\Delta S_{1}(\bar{l})}{\Delta I(\bar{l})}+\frac{\Delta S_{2}(\bar{l})}{\Delta I(\bar{l})} \\
& \quad=\frac{E\left\{\sum_{j=k M}^{k M+\bar{l}-1} \alpha_{j}^{2}\right\}}{\sum_{j=k M}^{k M+\bar{l}-1} I^{(j)}}+\frac{\pi \Omega}{2} \frac{\sum_{i=0}^{k M-1} \sum_{j=k M}^{k M+\bar{l}-1} d^{i+j}}{\sum_{j=k M}^{k M+\bar{l}-1} I^{(j)}} .
\end{aligned}
$$

Following the method used to prove Proposition 2, we can show that the first term in the above equation is a monotonic increasing function with respect to $\bar{l}$ when $1 \leq \bar{l} \leq M$. Now, we want to prove that the second term in (65) is also a monotonic increasing function. First, we can simplify it as

$$
\begin{aligned}
\frac{\pi \Omega}{2} \frac{\sum_{i=0}^{k M-1} \sum_{j=k M}^{k M+\bar{l}-1} d^{i+j}}{\sum_{j=k M}^{k M+\bar{l}-1} I^{(j)}}= & \frac{\pi \Omega d^{k M} \sum_{i=0}^{k M-1} d^{i}}{2 I^{(k M)}} \\
& \times \frac{\sum_{j=0}^{\bar{l}-1} d^{j}}{\sum_{j=0}^{\bar{l}-1} d^{2 j}}=g_{1} g_{2}(\bar{l})
\end{aligned}
$$

where $g_{1}=\left(\pi \Omega d^{k M} \sum_{i=0}^{k M-1} d^{i}\right) /\left(2 I^{(k M)}\right)$ and $g_{2}(\bar{l})=$ $\left(\sum_{j=0}^{\bar{l}-1} d^{j}\right) /\left(\sum_{j=0}^{\bar{l}-1} d^{2 j}\right)$. Since $g_{1}$ is independent of $\bar{l}$, we turn to demonstrate that $g_{2}(\bar{l})$ is a monotonic increasing function of $\bar{l}$. Note that

$$
\begin{aligned}
g_{2}(\bar{l}+1)-g_{2}(\bar{l}) & =\frac{\sum_{j=0}^{\bar{l}} d^{j}}{\sum_{j=0}^{\bar{l}} d^{2 j}}-\frac{\sum_{j=0}^{\bar{l}-1} d^{j}}{\sum_{j=0}^{\bar{l}-1} d^{2 j}} \\
& =\frac{\sum_{j=0}^{\bar{l}-1} d^{\bar{l}+j}\left(d^{j}-d^{\bar{l}}\right)}{\sum_{i=0}^{\bar{l}} d^{2 i} \sum_{j=0}^{\bar{l}-1} d^{2 j}}>0
\end{aligned}
$$

which indicates that $g_{2}(\bar{l})$ is a monotonic increasing function and so are $\left(\Delta S_{2}(\bar{l}) / \Delta I_{2}(\bar{l})\right)$ and $(\Delta S(\bar{l}) / \Delta I(\bar{l}))$.

\section{Proof of Proposition 3}

We first rewrite the codeword length $l$ as $l=k M+\bar{l}$ using (42). For $l \leq M$, i.e., $k=0$ and $l=\bar{l}$, the upper bound for the output SIR is $\bar{\nu}^{(M)}$ as suggested by Proposition 2. On the other hand, for $M<l \leq L$, Proposition 3 can be established by demonstrating that either one of the following statements is true.

1) If $\bar{\nu}^{((k+1) M)} \geq \bar{\nu}^{(k M)}, \max _{k M \leq l \leq(k+1) M} \bar{\nu}^{(l)}=$ $\bar{\nu}^{((k+1) M)}$

2) If $\bar{\nu}^{(k M)} \geq \bar{\nu}^{((k+1) M)}, \max _{k M \leq l \leq(k+1) M} \bar{\nu}^{(l)}=\bar{\nu}^{(k M)}$.

Here, we would like to prove the first statement under the assumption $\bar{\nu}^{((k+1) M)} \geq \bar{\nu}^{(k M)}$ holds. If $(\Delta S(\bar{l}) / \Delta I(\bar{l})) \leq$ $(S(k M) / I(k M))$ is true, Lemma 1 suggests that

$$
\begin{aligned}
\bar{\nu}^{(l)} & =\frac{S(k M)+\Delta S(\bar{l})}{I(k M)+\Delta I(\bar{l})} \leq \frac{S(k M)}{I(k M)} \\
& =\bar{\nu}^{(k M)} \leq \bar{\nu}^{((k+1) M)} .
\end{aligned}
$$

Otherwise, if $(\Delta S(\bar{l}) / \Delta I(\bar{l})) \geq(S(k M) / I(k M))$, we recall the fact that $(\Delta S(\bar{l}) / \Delta I(\bar{l})) \leq(\Delta S(M) / \Delta I(M))$ from Lemma 2, and then use Lemma 1 to get

$$
\begin{aligned}
\bar{\nu}^{(l)} & =\frac{S(k M)+\Delta S(\bar{l})}{I(k M)+\Delta I(\bar{l})} \leq \frac{S(k M)+\Delta S(M)}{I(k M)+\Delta I(M)} \\
& =\bar{\nu}^{(k+1) M} .
\end{aligned}
$$

The first statement is approved based on (67) and (68). The second statement can be proved similarly. Thus, it is omitted here.

\section{ACKNOWLEDGMENT}

The authors would like to thank the reviewers for their valuable comments which improved the quality of this paper and T. Lewis of the University of Southern California for valuable discussions on the pulse waveform design problem.

\section{REFERENCES}

[1] M. Z. Win and R. A. Scholtz, "On the energy capture of ultrawide bandwidth signals in dense multipath environments," IEEE Commun. Lett., vol. 2, no. 9, pp. 245-247, Sep, 1998.

[2] T. Strohmer, M. Emami, J. Hansen, G. Papanicolaous, and A. J. Paulraj, "Application of time-reversal with MMSE equalization to UWB communications," presented at the IEEE GLOBECOM, Nov. 2004.

[3] R. Esmailzadeh and M. Nakagawa, "Pre-RAKE diversity combining for direct sequence spread spectrum communications," presented at the IEEE ICC, May 1993. 
[4] S. Imada and T. Ohtsuki, "Pre-RAKE diversity combining for UWB systems in IEEE 802.15 UWB multipath channel," presented at the Joint UWBST \& IWUWBS, May 2004.

[5] K. Usuda, H. Zhang, and M. Nakagawa, "Pre-RAKE performance for pulse based UWB system in a standardized UWB short-range channel," presented at the IEEE WCNC, 2004.

[6] S. Niranjayan, A. Nallanathan, and B. Kannan, "Delay tuning based transmit diversity scheme for TH-PPM UWB: Performance with RAKE reception and comparison with multi RX schemes," presented at the Joint UWBST and IWUWBS, May 2004.

[7] D. Cassioli, M. Z. Win, and A. F. Molisch, "The ultra-wide bandwidth indoor channel: From statistical model to simulations," IEEE J. Sel. Areas Commun., vol. 20, no. 6, pp. 1247-1257, Aug. 2002.

[8] Revision of part 15 of the Commission's rules regarding ultra-wideband transmission systems, First Report and Order, ET Decoet 98-153, FCC 02-48, Federal Communications Commission (FCC), Feb. 14/Apr. 222002.

[9] Y.-L. Chao and R. A. Scholtz, "Weighted correlation receivers for ultra-wideband transmitted reference systems," presented at the IEEE GLOBECOM, Nov. 2004.

[10] J. R. Eoerster, "Channel modeling sub-committe report final," IEEE P802.15 WPAN P802.I5-02/490rJ-SG3a, Feb. 2003.

[11] A. F. Molisch, "Ultrawideband propagation channels-theory, measurement, and modeling," IEEE Trans. Veh. Technol., vol. 54, no. 5, pp. 1528-1545, Sep. 2005.

[12] J. Ibrahim, R. Menon, and R. M. Buehrer, "UWB signal detection based on sequence optimization for dense multipath channels," IEEE Commun. Lett., vol. 10, no. 4, pp. 228-230, Apr. 2006.

[13] C. Prettie, D. Cheung, L. Rusch, and M. Ho, "Spatial correlation of UWB signals in a home environment," presented at the UWBST02, May 2002.

[14] Y.-H. Chang, X. Yu, and C.-C. J. Kuo, "Techniques for received signal focusing in DSUWB systems," presented at the EEE VTC, Sep. 2005.

[15] A. J. Viterbi, CDMA:Principles of spread spectrum communication. Reading, MA: Addison-Wesley, 1995.

[16] T. P. Lewis and R. A. Scholtz, "An ultrawideband signal design with power spectral density constraints," presented at the 38th Asilomar Conf., Nov. 2005.

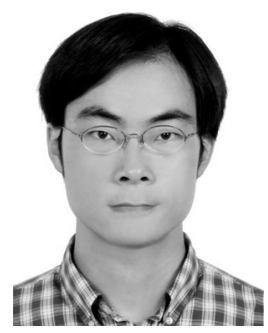

Yu-Hao Chang was born in Taichung, Taiwan, R.O.C., in 1976. He received the B.S. degree in electrical and control engineering from the National Chiao Tung University, Hsinchu, Taiwan, in 1998, and the M.S. degree in electrical engineering from the University of Southern California (USC), Los Angeles, in 2002. He is currently pursuing the Ph.D. degree at USC.

His current research interests focus on studying different signal process techniques for wireless communication systems.

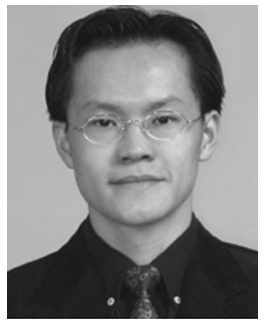

Shang-Ho Tsai (S'04-M'06) was born in Kaohsiung, Taiwan, R.O.C., in 1973. He received the B.S. degree in electrical engineering from Tamkang University, Taiwan, in 1995, the M.S. degree in electrical and control engineering from National Chiao-Tung University, Taiwan, in 1999, and the $\mathrm{Ph} . \mathrm{D}$. degree in electrical engineering from the University of Southern California, Los Angeles, in 2005.

From 1999 to 2002, he was with Silicon Integrated Systems Corporation (SiS), Taiwan, where he participated the VLSI design for DMT-ADSL systems. From 2005 to 2007, he was with MediaTek, Inc. (MTK), Taiwan, and participated the VLSI design for MIMO-OFDM systems. Since 2007, he has been with the Department of Electrical and Control Engineering, National Chiao Tung University, Hsinchu, Taiwan. His research interests include signal processing for communications, particularly the areas of multicarrier systems and space-time processing. He is also interested in VLSI design for these topics.

Dr. Tsai was awarded a government scholarship for overseas study from the Ministry of Education, Taiwan, for 2002-2005.

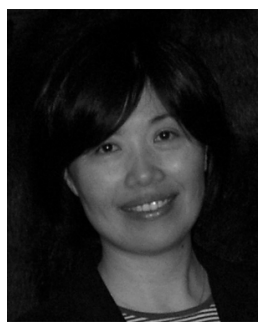

Xiaoli Yu received the Ph.D. degree in electrical engineering from the University of Southern California (USC), Los Angeles, in 1991.

In 1992, she was with the Space Computer Corporation, Santa Monica, CA, as a Research Scientist. From 1993 to 1997, she was a System Engineer and then a Research Instructor, both at the Molecular Imaging Science Center, Radiology Department, Keck School of Medicine, USC. Since 1998, she has been an Assistant Professor in the Departments of Radiology and Electrical Engineering, USC. Her research interests are in the areas of applying statistical signal processing and communication and information theory to wireless telecommunications systems and biological systems.

Dr. Yu received the 1999-2000 IEE Clarke-Griffiths Memorial Premium for the best paper in the IEE Proceedings on Radar, Sonar, and Navigation.

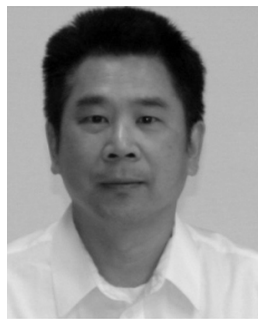

C.-C. Jay Kuo (F'99) received the B.S. degree from the National Taiwan University, Taipei, Taiwan, R.O.C., in 1980 and the M.S. and Ph.D. degrees from the Massachusetts Institute of Technology, Cambridge, in 1985 and 1987, respectively, all in electrical engineering.

He was a Computational and Applied Mathematics (CAM) Research Assistant Professor in the Department of Mathematics, University of California, Los Angeles, from October 1987 to December 1988. Since January 1989, he has been with the University of Southern California, Los Angeles, where he is currently a Professor of electrical engineering, computer science, and mathematics and Director of the Signal and Image Processing Institute. His research interests are in the areas of digital signal and image processing, multimedia compression, communication, and networking technologies. He is the coauthor of about 130 journal papers, 700 conference papers, and seven books.

Dr. Kuo is a Fellow of SPIE. He is the Editor-in-Chief for the Journal of Visual Communication and Image Representation and Editor for the Journal of Information Science and Engineering, LNCS Transactions on Data Hiding and Multimedia Security, and the EURASIP Journal of Applied Signal Processing. He received the National Science Foundation Young Investigator Award (NYI) and Presidential Faculty Fellow (PFF) Award in 1992 and 1993, respectively. 